\title{
Contract Enforcement: A Political Economy Model of Legal Development
}

\author{
Fali Huang* \\ Singapore Management University
}

In an effort to understand why the relative usage of relational and legal contracts differs across societies, this article builds a political economy model of legal development where legal quality of contract enforcement is a costly public good. It finds that legal investment tends to be too small under elite rule but too large under majority rule in comparison with the socially optimal level. Furthermore, elite rule, low legal quality, and high-income inequality may form a self-perpetuating circle that hinders economic development. In contrast to the conventional view, this article suggests that the often-observed association between heavy reliance on relational contracts and under development is most likely caused by the presence of elite rule rather than by a more collective-oriented culture per se because it is optimal for societies better at using relational contracts to start legal investment relatively late and to have lower quality of legal enforcement. (JEL O1, K49, H40, C72)

\section{Introduction}

Most economic transactions are prone to the risk of default by contracting partners, even though it is collectively beneficial for all relevant parties to act honestly. Understanding how to optimally manage such risk is thus essential to achieve efficient outcomes from voluntary exchanges. This problem is as old as human society and may become more severe over time as growing specialization in the economy generates more frequent and complex economic exchanges among agents. Various institutions have been created to solve this problem. A common enforcement method across different societies is to engage in bilateral relational contracts where a future stream of benefits is large enough to prevent short-sighted cheating today. In a multilateral environment such as a close-knit ethnic group or a social community, credible information of one's

\footnotetext{
*School of Economics, Singapore Management University, Singapore. Email: flhuang@smu. edu.sg.

The author is grateful to the Editor, W. Bentley MacLeod, a secondary editor, three anonymous referees, Michael Furmston, Andrew Phang, Daniel Rubinfeld, Noriyuki Yanagawa, seminar participants at Chinese University of Hong Kong, National University of Singapore, Singapore Management University, ISNIE (International Society of New Institutional Economics) Annual Conference in Reykjavik, Ronald Coase Institute Singapore Workshop, FEMES at Singapore, Econometrics Society World Congress in Shanghai, and International Conference on Institution and Economics in Japan for helpful comments.
}

The Journal of Law, Economics, \& Organization doi:10.1093/jleo/ews004

(C) The Author 2012. Published by Oxford University Press on behalf of Yale University. All rights reserved. For Permissions, please email: journals.permissions@oup.com 
past behaviors can be circulated at a low cost so that individual reputations can be developed and punishment carried out at the communal level. When it becomes more productive to trade with strangers outside one's community, the legal system is often relied upon to enforce contracts at the society level.

These different contract enforcement institutions have comparative advantages over each other and thus usually coexist in many societies. ${ }^{1}$ Their relative usage, however, differs across societies in an important way. The prevalence of legal contracts is typically associated with well-developed economies (such as the West), which exhibit high-quality legal systems and democratic political regimes, whereas a heavy reliance on informal relationships such as kinship and social communities is associated with developing countries that have the opposite characteristics. ${ }^{2}$ Such stylized differences lead naturally to the conjecture that a collective-oriented culture, which (in contrast to an individualistic culture) seems inherently prone to the usage of relational contracts, is the culprit behind both low legal quality and slow economic development (Weber 1954; North 1991; Greif 1994, 2006; North et al. 2000, 2009). This may be true for some countries; ${ }^{3}$ it is, however, difficult to reconcile with the experiences of recently developed East Asian economies under Confucian culture, namely Japan, Korea, Singapore, Hong Kong, and Taiwan (Landa 1981; Whyte 1996; Reed 2001).

This article develops a political economy model of legal development to account for why the relative usage of relational and legal contracts differs across societies. ${ }^{4}$ It finds that the existence of strong social networks per se (which is more likely in a collective culture) does not necessarily reduce overall welfare, though it may slow down the legal development process, since relational contracts have a comparative advantage in such an environment. ${ }^{5}$ However, if the intensive usage of relational contracts is accompanied by elite rule, then legal

1. See, for example, Landa (1981), Ellickson (1991), Bernstein (1992), Greif (1994, 2006), Whyte (1996), Durlauf and Fafchamps (2005), and Dixit (2009).

2. See North (1991), McMillan and Woodruff (1999), North et al. (2000), Fafchamps (2002), Johnson et al. (2002), and Greif (2006).

3. Latin America, for example, seems to fit these stylized facts. Compared with North America, the culture in Latin America is more collective-oriented (Portes 1994; North et al. 2000); the usage of informal contracts in Latin America is much higher, whereas the legal quality, the degree of institutionalized democracy during 1950-1995, the per capita GNP, and the average schooling level are much lower (De Soto 1989; Botero et al. 2004); the income inequality in Latin America is almost the highest among all countries (United Nations 2005).

4. Legal quality in this article refers not to the general quality of the legal system but only to the specific legal institutions that concern contract enforcement. Following related economics literature, the term "contract" in this article refers to any mutual agreement between relevant parties, where a "relational contract" is not backed up by law in any substantial sense and thus has to rely exclusively on beneficial future dealings to prevent cheating. In comparison, legal contracts rely explicitly on the third party, namely legal courts, for enforcement, even though future dealings or a relational aspect may still play some implicit role in mitigating cheating; this is consistent with the observation that in reality the relational aspect of exchange is also relied upon by those who use legal contracts (Macaulay 1963).

5. East Asia seems to fit this case compared with the benchmark case of the developed Western countries. 
quality is most likely to be inefficiently low, while income inequality is likely to be inefficiently high, both of which may hinder economic development. In other words, the presence of elite rule is a much stronger indicator than social structure or cultural orientation in assessing whether less reliance on legal contract enforcement is a barrier to economic development. In contrast, intensive reliance on legal contract enforcement is not necessarily welfare improving, especially when legal quality is determined by majority rule. ${ }^{6}$

Legal investment is treated in this article as a costly public good, whose provision is to a large degree determined by the amount of benefits resulting from using legal enforcement instead of relational contracts as well as by the political regime. $^{7}$ The analysis shows that relational contracts secure cooperation by promising future gains in an established relationship; the need to stay with current partners, however, makes individuals reluctant to do business with new partners even though they are more productive than the old ones. In contrast, legal contracts use an impersonal third party, the legal court, to deter cheating, and thus free agents from the burden of maintaining less productive relationships. ${ }^{8}$ As a result, the more productive new matches are relative to old ones, the higher the returns of using legal enforcement, and the larger the incentives to invest in the legal system. ${ }^{9}$

6. Magee et al. (1989) using data for about 35 countries show that a relatively large proportion of lawyers in a country's white-collar labor force leads to significant declines in economic performance (measured by GNP growth over the period 1960-80). Similar results are found by Murphy et al. (1991) based on a much larger data set. And it is well known that the number of lawyers per capita in the US is one of the highest in the world; there is also evidence that Americans collectively spend twice as much on civil litigation than they spend on new automobiles - more than any other industrialized country (Baye et al. 2005).

7. It is useful to note that, while informal contract enforcement at individual and communal levels seems to have been functioning spontaneously from early on, legal enforcement appears much later in history, and in general it needs intentional public investment to be established (Greif 2002, 2005). For instance, the establishment of legal courts, the development of legal codes and procedures, the training of judges, lawyers, and the police force are all needed for the legal system to work (Hadfield 2005).

8. This captures the insightful observation of Johnson et al. (2002): "Trust in existing suppliers may make entrepreneurs reluctant to purchase from new suppliers. . . The development of legal institutions brings indirect efficiency gains, by lowering entry barriers, in addition to direct efficiency gains through strengthening confidence in contracts."

9. It is useful to keep in mind that the relationship between relational contracts and legally enforced ones is much richer and more complicated than the model can capture. For example, in reality the relational aspect of exchange is also relied upon by those who use legal contracts (Macaulay 1963), which is also true in the model, and some legal rules may enhance the usage of relational contracts. However, such richness of mutual influence becomes much less salient than their distinction when one compares across societies where their relative usage varies dramatically. For example, settling disputes through a legalistic, adjudicatory system never became rooted in Chinese thinking, where for several thousand years the traditional perception was that the law was primarily criminal and thus going into litigation was perceived as a disgraceful and humiliating process that should be avoided at all costs; as a result, contract law was ignored throughout Chinese legal history until recently (Peng 2000). As the main purpose of this article is to understand crosssociety variation in the usage of legal contract enforcement, such abstraction of the distinction between relational and legal enforcement seems to be warranted, otherwise we may get lost in details and miss the big picture. 
A direct implication is that a society would start to invest in legal enforcement only when the benefits of doing so become high enough relative to the default choice of using relational contracts. So across societies, those more suitable for the usage of relational contracts (e.g., those with a more homogeneous population, better organized communities, or a collective culture) tend to start legal investment late and have lower legal quality. In other words, the socially optimal pace of legal development may vary across societies because the comparative advantages of relational contract enforcement may differ due to exogenous reasons. This suggests that a higher quality legal system is not always better for development, and a collective culture is not necessarily bad.

When individuals have heterogeneous returns and thus conflicting interests in improving legal quality, a political economy model is needed to examine how legal investment is determined. Specifically, this article observes that the traditional rich elite, who by definition are richer than others at times when legal contract enforcement is not available or weak, must have enjoyed higher gains in using relational contracts. ${ }^{10}$ But this means they would benefit relatively less from legal enforcement, whereas the less privileged masses stand to gain more from having a competent legal system. ${ }^{11,12}$ So it is not surprising to see that, if the rich elite are politically dominant and choose legal investment

10. Mapping from theory to reality, it is useful to note that the traditional elite are often endowed with land and other natural resources, which are relatively stable sources of income, and thus their major business transactions are conducted mostly through relational contracts with similar elite families (Adams and Brownsword 2007; North et al. 2009).

11. This result is consistent with the comment of Pipes (1995:289): "Those in power have no need of courts and laws to have their way; it is the poor and the weak who do. Anyone who doubts this proposition has only to compare the general condition and the sense of security of the lower orders in areas with weak legal traditions, as for example south-east Asia, with those like western Europe and the United States where they are deeply entrenched." The claim that the masses in general have more to gain from a competent legal system compared with a dysfunctional one than the elite is not contradictory to the observation that a poor individual may benefit less than an elite even in a highly developed legal system.

12. Note that the elite are composed of a rich minority, whereas the masses that favor legal enforcement are the majority of the population and not necessarily the poorest sector of a society. For example, North et al. (2009:93) estimate that the landed elite group in England was only about $0.5 \%-1 \%$ of the whole population between 1200 and 1600 , which implies that the masses contained at least $99 \%$ individuals in society. On a related note, legal enforcement in the model proposed in the present article comes not from litigation per se but, instead, from its absence, because in the equilibrium everyone cooperates and thus there is no need to have resort to litigation. In other words, the implicit background role of law is much more crucial for legal enforcement to work than expensive litigation, which occurs only off the equilibrium path and thus should be relatively rare. Having said that, it must be acknowledged that the ability to defend one's interests in litigation when it indeed happens remains important. Though conceptually the masses must have adequate access to the legal system for any law to be effective, which is also feasible, whether this is actually achieved is an important condition for the mechanism of the model to work. In this regard, even in the context of litigation, many cases are settled and cases that in fact go for full trial are, to that extent, relatively rare. Finally, the availability of legal aid and (in certain countries at least) contingency fee or conditional fee arrangements do mitigate (to some extent at least) the problem of costs and the concomitant obstacles with regard to access to justice. 
to maximize their own welfare, legal development tends to be slower than the socially optimal level, whereas the opposite is true if the masses are politically dominant. In other words, legal investment tends to be too large under majority rule and too small under elite rule, and thus legal quality is often higher in democratic societies than in others.

Another finding is that income inequality falls when legal quality improves; the reason is that a better legal system, by providing all agents with more equal access to new trade opportunities, makes the quality of their initial matches and endowment less important and thus dampens the income gaps between the traditional elite and the rest. ${ }^{13}$ Furthermore, the links between elite rule, low legal quality, and high inequality can be mutually reinforcing when the political power of a group is affected by its collective economic strength (Engerman and Sokoloff 2002, 2005). ${ }^{14}$

This article contributes to the literature of contract enforcement institutions by formally endogenizing legal quality in a political economy model of legal development. ${ }^{15}$ Many studies examine comparative advantages of different enforcement institutions and how they may be affected by exogenous changes. ${ }^{16}$ Though the endogenous determination of enforcement institutions is also featured in a few studies, the focus and approach are different in this article. For example, Greif (2005) stresses the importance of coercive constraint institutions, whereas this article adopts a different angle by examining the effects of heterogeneous returns among agents from using relational and legal contracts. ${ }^{17}$

From a broader perspective, this article is also connected to studies demonstrating the harmful effects of high income-inequality on institutions, where the privileged elite shape institutions to suit their narrow interests. ${ }^{18}$ This

13. This is consistent with the positive correlation between high-income inequality and intensive usage of informal relational contracts (Chong and Gradstein 2007b).

14. In this case, we have a self-perpetuating circle of economic, political, and legal conditions: A highly unequal endowment distribution gives rise to elite rule, which slows down the legal development, and low legal quality in turn helps preserve high-income inequality. Similar arguments suggest the existence of the opposite circle containing low-income inequality, democracy, and high legal quality.

15. See MacLeod (2007) and Dixit (2009) for systematic surveys and synthesis of related work.

16. Examples include better legal quality (Cooter and Landa 1984; Sobel 2006; Besley and Ghatak 2009), expanding trade (Dixit 2003; Greif 2006), access to market (Kranton 1996a) and financial crisis (Li 2003).

17. Besley and Ghatak (2009) introduce a costless legal reform by the social planner to enhance the use of formal collateral, whereas Dhillon and Rigolini (2011) endogenize enforcement institutions by allowing firms to bribe legal courts and consumers to share information. To the best of my knowledge, I am not aware of similar attempts to use a political economy model to study legal development in the legal scholarship; for a possible explanation see, for example, Harris (2003).

18. See, for example, Glaeser et al. (2003), Sonin (2003), Hoff and Stiglitz (2004), Acemoglu and Robinson (2006b, 2008), Chong and Gradstein (2007a). 
article differs from these studies in several aspects. First, a key difference is in the exact source of inefficiency. A common result in the related literature is that the rich elite can take advantage of lawlessness by engaging in rentseeking activities or corruption, which gives rise to economic stagnation and inefficient institutional change. In this article, the inefficiency is not caused by rent-seeking activities, but by genuine differences in benefits from improving legal quality, where the traditional elite have comparative advantages in using relational contracts. ${ }^{19}$ Second, and more importantly, this leads to a new insight that has not been noticed in the literature: Inefficient legal development not only occurs under elite rule but also happens under majority rule; that is, while elite-ruled societies tend to under-invest in legal contract enforcement, democratic societies tend to overinvest in it. ${ }^{20}$ Finally, none of these studies endogenizes the development of legal contract enforcement or examines its relationship with relational contracts and social communities.

This article proceeds as follows. The political economy model is set up in the next section. Relational and legal contracts are analyzed in section 3 , whereas legal quality is endogenized in section 4. Some extensions and relevant examples are provided in section 5. The final section concludes the article. All technical proofs are in the Appendix.

\section{The Model}

There is a continuum of agents with a unit mass who live infinitely. A small proportion $r$ of agents are the elite, each endowed with wealth $w_{e}$ and education $h_{e}$, while the others belong to the masses, each endowed with a smaller wealth $w_{m}$ and lower education $h_{m}$, where $w_{e}>w_{m}>0$ and $h_{e}>h_{m}>0$.

The model contains two phases. In the first phase, the legal quality $q$ of contract enforcement is determined through a political process; it is taken as given in the second phase, when agents match with each other into pairwise partnerships to carry out projects.

Legal Investment. The legal quality $q$ of contract enforcement is chosen to maximize the total welfare of the politically dominant interest group, whereas the cost is equally shared among all agents. The cost function of legal investment is $C(q)$, where $C^{\prime}(q)>0$ and $C^{\prime \prime}(q)>0$. Without any investment, the initial legal quality is zero. The cost of improving legal quality is presumably composed of writing the legal rules and training judges, lawyers, the police

19. The point that the rich can do better in relational contracts is not new; Banerjee and Newman (1993), for example, provide a mechanism linking relational contracts and inequality; they do not, however, consider the political economy of contract enforcement as this article does.

20. A case of overusing legal contract enforcement is shown in Kranton and Swamy (1999), and in general, it seems to be consistent with the recent evidence of declining social capital in the US (see, among others, Putnam 1995 and Durlauf and Fafchamps 2005). Though potential drawbacks of democracy in comparison to oligarchy are also discussed in Acemoglu (2008), the bad result there is higher taxes, a well-known consequence under majority rule, not the inefficiency of institutions as highlighted in this article. 
force, etc. Though these details are not explicitly modeled in the article, their overall effectiveness is indicated by $q$.

Repeated Matching Game. The second phase of the model can be described as a repeated matching game. ${ }^{21}$ Agents match with each other to play a twoplayer repeated game, which can be interpreted as engaging in a business partnership. In each period, a match continues if both players agree to participate, and it breaks up if either one wishes to do so.

Stage Game. In a match, agents play the prisoner's dilemma (PD) described in Table 1. When both agents cooperate in the project, each gets a return of $a>0$, which represents the gains from trade for both players. If one agent cooperates but the other defects, the cooperator gets a negative payoff $-d<0$ whereas the cheater gets a higher return $a+b$, where $b>0$ represents the temptation of cheating; cheating is bad for the total surplus, which is captured by the assumption $2 a>a+b-d>0$. If both agents defect, then each gets a return of zero, which is the normalized return of going autarky.

Return on Learning in Established Matches. After a partnership is formed for some time, the gains from trade may be improved through learning-bydoing, and the improvement scale increases in the education levels of the two partners. In particular, the return from the partnership becomes $a\left(1+g\left(h_{1}, h_{2}\right)\right)$ from the second period onward after the partnership is formed, where $g_{1}, g_{2}>0$ and $g_{12} \geq 0$. So agents prefer to have a more educated partner, which will lead to perfect sorting such that elite agents match among themselves, as do nonelite agents. ${ }^{22}$ It is thus useful to denote $g_{e} \equiv g\left(h_{e}, h_{e}\right)$ and $g_{m} \equiv g\left(h_{m}, h_{m}\right)$; it is clear that $g_{e}>g_{m}$ holds based on the properties of $g\left(h_{1}, h_{2}\right)$ and $h_{e}>h_{m}$. Since the temptation of cheating remains $b$ as before, the payoff for the cheater in the stage game becomes $a\left(1+g_{i}\right)+b$ in an established match where $i \in\{e, m\}$.

Increase in Outside Opportunities. In each period, the gains from trade in a new match stay the same at $a$ with probability $\rho$, where $\rho \in(0,1)$. With probability $1-\rho$, there is an exogenous shock that increases the gains from trade for newly formed partnerships from $a$ to $a(1+\varepsilon)$ for the first $N$ periods, where $\varepsilon>0$, after which the gains from trade go back to normal; that is, they

Table 1. The Stage Game: A Prisoner's Dilemma

\begin{tabular}{llll}
\hline & \multicolumn{2}{c}{ Agent 2 } \\
\cline { 3 - 4 } & & Cooperate & Defect \\
\hline Agent 1 & Cooperate & $(a, a)$ & $(-d, a+b)$ \\
& Defect & $(a+b,-d)$ & $(0,0)$ \\
\hline
\end{tabular}

21. Some parts of this repeated game are similar to Sobel (2006).

22. This is meant to capture the privileges enjoyed by the elite; alternative ways of modeling, for example, by assuming that the elite have better connections or own better projects, would yield similar results. 
become $a\left(1+g_{i}\right)$. This is meant to capture the influence of new trading opportunities that are exogenously determined and beyond the control of agents, where $\varepsilon$ indicates the surplus from outside opportunities that cannot be reaped if one stays in the old partnership. Without much loss of generality, we assume $N=1$ to simplify the analysis. ${ }^{23}$ As the shocks do not change the temptation of cheating $b$, the payoff for cheating in the stage game becomes $a(1+\varepsilon)+b$ when the gains from trade are $a(1+\varepsilon)$.

Information. There is no information transmission across matches. Agents know the quality of their current match and the past actions of their own and their partners within the matches. They cannot access information about the past actions of any other agents. Since the population of agents is large, we neglect the possibility that any two agents have met before. Unmatched agents can find a new partner without cost. ${ }^{24}$

Strategy and Equilibrium. In each period of a match, an agent's strategy specifies an action in the above PD game, that is, to cooperate or to defect, followed by a decision on whether to continue or to terminate the partnership. Agents choose strategies to maximize the discounted sum of their stage-game payoffs, net of contracting costs, if any, where the common discount factor is $\delta \in(0,1)$. This article focuses on subgame perfect equilibrium (SPE) outcomes, where an agent discontinues a partnership only if doing so gives him a better payoff than otherwise.

Relational Contract. In particular, we study two types of enforcement institutions (which are SPEs) that enable agents to cooperate. One is a long-term relational contract that demands both agents to always cooperate and to continue the partnership regardless of exogenous shocks, and if any agent defects, it dissolves automatically at no cost to either agent. To deter cheating, each agent in the partnership has to incur a sunk cost $R$ up-front, which cannot be recovered once the relationship stops. ${ }^{25}$ The expenditure $R$ can be interpreted as the cost of building the relationship, such as exchanging gifts or bonds, or spending time and resources participating in social activities, which are quite common procedures for initiating business relationships in many societies. This means that, if an agent breaks the current partnership and forms a new one with a stranger, he must pay $R$ again for the new partnership, otherwise he has to face the risk of being cheated.

Legal Contract. The other type of enforcement is to sign a short-term formal legal contract that mandates cooperation during the match and punishes cheating but allows agents to break up when a new match becomes more

23. Assuming $N>1$ or $N=+\infty$ will not change the main qualitative results.

24. When there is an endogenous matching cost, the qualitative results remain unchanged (see Sobel 2006). The information transmission assumption will be relaxed in section 5.2 when social communities are discussed.

25. The observation that imposing costs at the beginning of a relationship can lead to efficiency gains is also made by Carmichael and MacLeod (1997) and Kranton (1996b). 
productive. ${ }^{26}$ If a pair of players each takes a cost $c$ to write a contract, the court identifies cheating when it occurs with probability $Q(c, q)$, where $q$ denotes legal quality as already mentioned. The related cost of writing and using legal contracts includes, for example, the effort to specify and follow appropriate procedures in order to produce adequate evidence for the legal court to verify whether cheating has happened and to carry out a possible remedy for breach; a higher cost may imply a greater amount of documents or information to be presented or exchanged before payment is to be made or goods are to be delivered, which should increase the probability of breach being verified and punished by the legal court. Similarly, such a probability is also higher when the legal system is more effective. So we assume $Q_{c}, Q_{q}>0$, $Q_{c q} \geq 0$, and $Q(0, q)=Q(c, 0)=0$. When cheating is verified by the court, the defector has to give his unjust gain, the payoff $a(1+\tau)+b$, to his partner, where $\tau \in\{0, g, \varepsilon\}$ indicates the three possible levels of productivity in a partnership. ${ }^{27}$

Timing. The timing of this repeated game can be summarized as follows. Players form pairs with each other through random matching within their respective groups and, subject to mutual agreement, partners choose to adopt either a relational contract or a legal contract, and then behave accordingly. A match breaks up automatically once unexpected cheating occurs or at least one player decides to dissolve it. Players exiting from an old relationship form new matches and then the same action sequence described above follows.

\section{Relational and Legal Contracts}

The model is analyzed using backward induction. This section focuses on the second phase of the model, namely the repeated matching game, while taking the legal quality $q$ as given; it examines how agents adopt different contracts that are indeed subgame perfect equilibria under certain conditions.

\subsection{Long-Term Relational Contracts}

Suppose a pair of type $i$ agents, where $i \in\{e, m\}$, choose a long-term relational contract that does not dissolve due to exogenous shocks. Since the relational contract demands cooperation in all periods, where the return is $a$ in the first period and $a\left(1+g_{i}\right)$ afterward, the value of such a new match to each partner is denoted by $V_{y i}$ where

26. In addition to these two types of contracts that are the focus of this article, it is possible to have other contract formats such as short-term relational contracts and long-term legal contracts, which are, however, less commonly used. An earlier version of this article includes them and the main results are similar. It is also useful to clarify that though strong words like "cheating" and "punishment" used in this article are applicable in the context of a PD, in other cases of contract law they should be read as "breach of contract" and "remedy" that carry no moral judgment.

27. This remedy for breach is a restitutionary award to the innocent party (Furmston 2006). Other reasonable assumptions about the court's decision, such as the usual expectation damage measures or a reliance measure, will not alter the main results. 
$V_{y i}=a+\frac{\delta a\left(1+g_{i}\right)}{1-\delta}$.

Similarly, when the initial gain from trade is $a(1+\varepsilon)$ followed by periods with a return of $a\left(1+g_{i}\right)$, the value of a new match is denoted by $V_{n i}$, where $V_{n i}=V_{y i}+a \varepsilon$ holds. To prevent cheating, both agents have to incur a relationship-building $\operatorname{cost} R_{i}$, which turns out to be the same regardless of the level of the initial returns.

Let's check possible one-shot deviations when the initial gain from trade is $a$. Deviations may happen either in a new match or when the match becomes established. In a new match, a player gets $V_{y i}-R_{i}$ if he cooperates; if he defects, his payoff is $a+b-R_{i}+\delta E V_{i}$, where $a+b$ is the current payoff from defecting, and the continuation value $E V_{i} \equiv \rho V_{y i}+(1-\rho) V_{n i}-R_{i}$ is obtained by forming a new match in the next period: with probability $\rho$, the gain from trade remains the same so the net value of a new match is again $V_{y i}-R_{i}$, whereas with probability $1-\rho$ the gain from trade increases to $a(1+\varepsilon)$ so the value of a new match is $V_{n i}-R_{i}$. Cheating is not profitable when cooperation yields a higher return than defecting, which is the case when

$R_{i} \geq b / \delta-a g_{i}+(1-\rho) a \varepsilon$

holds. It can be shown that this same condition also prevents cheating in an established partnership and when the initial gain from trade is $a(1+\varepsilon) .{ }^{28}$

To maximize the net value of the match, the relationship-building cost should be set at

$R_{i}^{*} \equiv b / \delta-a g_{i}+(1-\rho) a \varepsilon$,

which is obtained from equation (1) at equality, and no agent has any incentive to defect. $R_{i}^{*}$ is thus the minimum cost of using the long-term relational contract to achieve cooperation. It is larger when the temptation of cheating $b$ is higher, when agents are less patient (i.e., when $\delta$ is lower), when the old partnership is less productive ( $g_{i}$ lower), and when outside opportunities are better ( $a \varepsilon$ higher) and arrive more frequently $((1-\rho)$ larger).

Another condition for the long-term relational contract to be a SPE is that it must be desirable to continue the old match even when a new match becomes more productive. The net value of starting a new match is $V_{n i}-R_{i}^{*}$ when the gain from trade is $a(1+\varepsilon)$, while the value of continuing with the old match is $a\left(1+g_{i}\right) /(1-\delta)$. So it is optimal for agents to keep the old match when $a\left(1+g_{i}\right) /(1-\delta) \geq V_{n i}-R_{i}^{*}$, which implies

$a \varepsilon \leq R_{i}^{*}+a g_{i}$.

This condition says that, when the productivity increase in new trade opportunities (indicated by $a \varepsilon$ ) is not large enough to compensate for the loss of productivity gain $\left(a g_{i}\right)$ from learning-by-doing in the old match and the cost

28. The formal proof is in the Appendix. 
$\left(R_{i}^{*}\right)$ of building a new relationship, it is optimal to stay in the current match. When $R_{i}^{*}$ is replaced by equation (2), this condition becomes

$\varepsilon \leq b / \delta \rho a$,

where $b / \delta \rho a$ is the threshold level of productivity shock, below which neither type of agent has an incentive to dissolve the match. Thus, we have proved the following results.

Lemma 1 When outside opportunities are low $(\varepsilon \leq b / \delta \rho a)$, the long-term relational contract is a SPE for both elite and nonelite agents, whereas the elite benefit more from using relational contracts than the others (i.e., $\left.V_{y e}-R_{e}^{*}>V_{y m}-R_{m}^{*}\right)$.

This lemma suggests that it is optimal for agents to engage in long-term relational contracts as long as the productivity gains in new trade opportunities are not too large compared with the forgone return on learning in an established match and the new relationship-building cost. And by having higher returns in established partnerships $\left(g_{e}>g_{m}\right)$, the elite have larger gains from using longterm relational contracts than the others.

\subsection{Short-Term Legal Contracts}

Suppose a pair of agents of type $i \in\{e, m\}$ choose to adopt a short-term legal contract that punishes cheating but allows agents to break up at the beginning of a period when a new match is more productive. Let $\hat{V}_{y i}$ and $c_{i}^{*}$ denote, respectively, the value and the optimal cost of using a legal contract to deter cheating when the initial return is $a$. Using similar arguments as in the above analysis of relational contracts to check for one-shot deviations, we get the following results.

Lemma 2 The optimal legal $\operatorname{cost} c_{i}^{*}$ is higher when the legal quality $q$ is lower and when the established match is less productive ( $g_{i}$ is lower), whereas it is independent of outside opportunities $\varepsilon$.

Note that, while the relationship-building $\operatorname{cost} R_{i}^{*}$ increases with new trade opportunities $\varepsilon$, the $\operatorname{cost} c_{i}^{*}$ of using legal contracts is independent of it because legal enforcement allows the old match to dissolve once a new match becomes more productive. In other words, agents adopting short-term legal contracts do not need to face the pressure of maintaining the relatively less productive old match.

For a short-term legal contract to be a SPE, it must be desirable to break up the old match when a new match becomes more productive, which is indeed true when

$a \varepsilon \geq c_{n i}^{*}+a g_{i}$ 
holds, where $c_{n i}^{*}$ denotes the legal cost when the initial return is $a(1+\varepsilon)$. This condition is parallel to equation (3) in the case of relational contracts; it says that when the new trade opportunities (indicated by $a \varepsilon$ ) are large enough to compensate for the $\operatorname{cost} c_{n i}^{*}$ of writing a new legal contract and the loss of return on learning $\left(a g_{i}\right)$ in the old match, it is optimal to break up the current match and form a new one. These are formally proved in the following lemma.

Lemma 3 When outside opportunities are high $\left(\varepsilon \geq \varepsilon_{i}\right)$, the short-term legal contract is a SPE for an agent of type $i$, where the threshold level $\varepsilon_{i} \equiv g_{i}+c_{n i}^{*} / a$ is derived from equation (4). Legal contracts enable agents to take advantage of new opportunities more often than relational contracts but they are less likely to be used by the elite (because $\left.\varepsilon_{m}<\varepsilon_{e}<b / \delta \rho a\right)$.

This lemma suggests that it is optimal for agents to engage in short-term legal contracts once the outside opportunities are large enough compared with the legal cost and the forgone benefit of learning in an established match. Given that the threshold level of new opportunity is higher $\left(\varepsilon_{e}>\varepsilon_{m}\right)$ for the elite, they are less willing to adopt short-term legal contracts than others, where the underlying reason is again due to their higher returns in established partnerships $\left(g_{e}>g_{m}\right)$. Comparing the two types of contracts, the condition $\varepsilon_{m}<\varepsilon_{e}<b / \delta \rho a$ suggests that legal contracts enable agents to break up old matches more often than relational contracts (with threshold $b / \delta \rho a$ derived above) in order to take advantage of new opportunities.

\subsection{Comparison between Relational and Legal Contracts}

Recall that, for an agent of type $i \in\{e, m\}$, the net value of using a long-term relational contract is $V_{y i}-R_{i}^{*}$ and that of using a short-term legal contract is $\hat{V}_{y i}-c_{i}^{*}$. So the benefit of switching from relational to legal contracts is the difference between these two net values: $\pi\left(q ; \varepsilon, g_{i}\right) \equiv\left(\hat{V}_{y i}-c_{i}^{*}\right)-\left(V_{y i}-R_{i}^{*}\right)$, which after some algebra becomes

$$
\begin{aligned}
\pi\left(q ; \varepsilon, g_{i}\right)= & (1-\rho)(a \varepsilon-b / \delta \rho) \\
& +\frac{\delta(1-\rho)}{1-\delta}\left(a \varepsilon-a g_{i}-c_{n i}^{*}\right)+\frac{a+b}{\delta \rho} Q\left(c_{i}^{*}, q\right) .
\end{aligned}
$$

Its properties and implications are summarized below.

Lemma 4 The relative benefit of using legal contracts is larger and thus relational contracts are used less when outside opportunities $\varepsilon$ are larger, when the legal quality $q$ is higher, and when the return on learning $g_{i}$ is lower. For any given $\varepsilon$ and $q$, since the elite enjoy higher gains from established matches than others $\left(g_{e}>g_{m}\right)$, they benefit less from switching to legal contracts. 
The intuition is as follows. Better outside opportunities make it more worthwhile to break up the old match since a new partnership promises higher gains from trade. The benefit is also larger when the legal quality $q$ is higher because the legal cost of forming new matches decreases in $q$. Since the elite have comparative advantages in using long-term relational contracts due to $g_{e}>g_{m}$, they are less willing to adopt legal contracts than the nonelite. This is the main insight that is underlying the different incentives for legal investment.

\section{Investment in Legal Quality}

This section analyzes the first phase of the model where the legal quality $q$ is endogenized in a political economy context. Specifically, the politically dominant interest group chooses the level of legal quality to maximize its joint welfare while taking into consideration the effect of legal quality on its expected returns in the subsequent repeated matching game. ${ }^{29}$ Without any investment, the initial legal quality is zero and thus all agents use relational contracts. So the legal investment, if ever made, has to be large enough to make agents willing to shift from relational to legal contracts.

\subsection{Socially Optimal Legal Investment}

The socially optimal level of legal investment is determined to maximize the aggregate welfare of all agents. The total benefit of increasing the legal quality from 0 to $q$ is $r \pi\left(q ; \varepsilon, g_{e}\right)+(1-r) \pi\left(q ; \varepsilon, g_{m}\right)$ if all agents shift from long-term relational contracts to short-term legal contracts, which is the focus of the following analysis. ${ }^{30}$ The total investment $\operatorname{cost} C(q)$ is equally shared among all agents. So the optimal legal quality $q^{s}$ is defined by

$q^{s} \equiv \arg \max _{q}\left\{r \pi\left(q ; \varepsilon, g_{e}\right)+(1-r) \pi\left(q ; \varepsilon, g_{m}\right)-C(q), 0\right\}$.

Since the benefits of using legal contracts are strictly increasing in the outside opportunities $\varepsilon$, there must exist a unique level $\varepsilon^{s}$ such that

$r \pi\left(q^{s} ; \varepsilon^{s}, g_{e}\right)+(1-r) \pi\left(q^{s} ; \varepsilon^{s}, g_{m}\right)-C\left(q^{s}\right)=0$.

So $\varepsilon^{s}$ is the socially optimal threshold level of $\varepsilon$, above which legal investment starts.

Proposition 1 When outside opportunities are low $\left(\varepsilon \leq \varepsilon^{s}\right)$, it is socially optimal to have no legal investment. Legal development starts only when

29. Here the legal quality refers to the actual quality of legal enforcement, not to the quality of the rule book, which can be amended without much cost.

30. It is also possible that the legal quality is only high enough for the nonelite agents to switch to legal contracts while the elite still use relational contracts, in which case the total benefit of legal investment is $(1-r) \pi\left(q ; \varepsilon, g_{m}\right)$. As the main results are similar, this case is omitted to simplify the exposition. 
outside opportunities are high enough $\left(\varepsilon>\varepsilon^{s}\right)$, where $\varepsilon^{s}$ increases while $q^{s}$ decreases with return on learning $g_{i}$.

This proposition suggests that the threshold level of outside opportunities, $\varepsilon^{s}$, for a society to start legal investment is higher and the optimal legal quality $q^{s}$ is lower when the productivity of established matches represented by $g_{i}$ is higher. If $\varepsilon$ is drawn from some exogenous distribution $F(\cdot)$, the probability of investing in legal quality is $1-F\left(\varepsilon^{s}\right)$, which is lower if $g_{i}$ is higher. So in the socially optimal case, a society is less likely to invest in legal quality and invests less (if ever invested) when long-term relational contracts are more effective in achieving cooperation.

\subsection{Legal Investment with Exogenous Political Systems}

As the benefits from legal investment differ across agents, which has become clear in the preceding analysis, political conflicts may play an important role in the determination of legal quality. Suppose the political system is determined exogenously, where a society is either under elite rule or under majority rule, and legal quality is chosen to maximize the welfare of the politically dominant group.

Legal Investment under Elite Rule. Under elite rule, the elite group is dominant and hence will choose a legal investment level to maximize its own welfare. The total benefit for the elite group from increasing legal quality from 0 to $q$ is $r \pi\left(q ; \varepsilon, g_{e}\right)$ while the cost it has to pay is $r C(q)$, since the total cost $C(q)$ is shared among all agents where the elite group is of $r$ proportion. ${ }^{31}$ So the optimal legal quality $q_{e}^{*}$ under elite rule is

$q_{e}^{*} \equiv \arg \max _{q}\left\{r \pi\left(q ; \varepsilon, g_{e}\right)-r C(q), 0\right\}$.

Since $\pi\left(q ; \varepsilon, g_{e}\right)$ is strictly increasing in $\varepsilon$, there must exist a unique level $\varepsilon^{e}$ such that

$\pi\left(q_{e}^{*} ; \varepsilon^{e}, g_{e}\right)-C\left(q_{e}^{*}\right)=0$.

So the elite ruling society starts legal investment only when $\varepsilon>\varepsilon^{e}$, in which case the legal quality $q_{e}^{*}$ is uniquely determined by

$\pi_{1}\left(q_{e}^{*} ; \varepsilon, g_{e}\right)-C^{\prime}\left(q_{e}^{*}\right)=0$.

31. The legal investment cost $C(q)$ can be paid by tax revenues generated from the population. Here, it is assumed that each agent pays the same amount of tax. Alternative cost-sharing methods should not change the main results. For example, in the extreme case where the elite agents are required to pay nothing for legal investment, they will not start it unless $\pi\left(q ; \varepsilon, g_{e}\right) \geq 0$, but the socially optimal legal development may start even when $\pi\left(q ; \varepsilon, g_{e}\right)<0$ is true. And more importantly, there is always an opportunity cost of improving legal quality, since the tax revenues can be used in other ways to increase the elite's utility. In this sense, legal investment is always costly from the elite's perspective. 
Proposition 2 Under elite rule, the threshold outside opportunity $\varepsilon^{e}$ to start legal investment is higher and the legal quality $q_{e}^{*}$ is lower than in the socially optimal case, and the more so when return on learning $\mathrm{g}_{\mathrm{e}}$ is higher.

This proposition suggests that the elite are less likely to invest in legal quality and invest less (if ever invested) when they are relatively more productive in established partnerships. And compared with the socially optimal case, legal quality is lower and the threshold productivity shock needed to start legal investment is higher under elite rule. So legal development is slower under elite rule than in the socially optimal case, and thus agents are more likely to use relational contracts and less likely to use legal contracts.

Legal Investment under Majority Rule. Under majority rule, the masses are politically dominant and thus choose a level of legal quality to maximize their overall welfare $(1-r) \pi\left(q ; \varepsilon, g_{m}\right)-(1-r) C(q)$. The same results will go through if the median voter of the population chooses the optimal legal investment to maximize his own welfare, since all nonelite agents are identical and they constitute the majority. So legal quality under democracy with majority voting will be the same. The analysis is similar to that under elite rule. The optimal legal quality $q_{m}^{*}$ under majority rule is uniquely determined by

$\pi_{1}\left(q_{m}^{*} ; \varepsilon, g_{m}\right)-C^{\prime}\left(q_{m}^{*}\right)=0$

when $\varepsilon>\varepsilon^{m}$, and is zero otherwise, where the threshold outside opportunity $\varepsilon^{m}$ satisfies

$\pi\left(q_{m}^{*} ; \varepsilon^{m}, g_{m}\right)-C\left(q_{m}^{*}\right)=0$.

Proposition 3 Under majority rule, the threshold outside opportunity $\varepsilon^{m}$ to start legal investment is lower and the legal quality $q_{m}^{*}$ is higher than in the socially optimal case, and the more so when return on learning $g_{m}$ is lower.

This proposition suggests that, compared with the socially optimal case, legal quality under majority rule is larger and the threshold productivity shock needed to start legal investment is lower. In other words, a society under majority rule may overinvest in legal quality to a level higher than what is socially optimal. This is not surprising, since the masses, who are more disadvantaged under long-term relational contracts than the elite, can enjoy more benefits from utilizing new trade opportunities with legal contracts and hence have more incentives to improve the legal system.

Comparison between Political Regimes. Depending on the level of $\varepsilon$, legal development may differ across political regimes. There are three possible scenarios, which are illustrated in Figure 1 and analyzed below. 


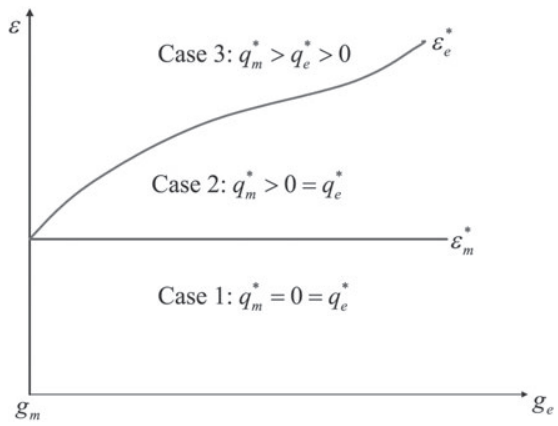

Figure 1. Legal Investment under Different Political Regimes.

Case 1: Small Outside Opportunities $\left(\varepsilon \leq \varepsilon^{m}\right)$. There is no legal investment in any political system so that $q_{m}^{*}=0=q_{e}^{*}$ and only long-term relational contracts are used. The income inequality, as represented by the income gap $G_{1}$ between the two types of agents, is the largest, where

$$
G_{1} \equiv V_{y e}-R_{e}^{*}-\left(V_{y m}-R_{m}^{*}\right)=a\left(g_{e}-g_{m}\right) /(1-\delta)
$$

is derived from results in the last section.

Case 2: Medium Outside Opportunities $\left(\varepsilon^{m} \leq \varepsilon \leq \varepsilon^{e}\right)$. There is still no legal investment under elite rule, but the society under majority rule will start to invest in the legal system so that $q_{m}^{*}>0=q_{e}^{*}$. As a result, agents under majority rule adopt legal contracts and thus can take advantage of the new trade opportunities that are more productive, which decreases income inequality to ${ }^{32}$

$$
G_{2}\left(q_{m}^{*} ; \varepsilon\right) \equiv \hat{V}_{y e}-c_{e}^{*}-\left(\hat{V}_{y m}-c_{m}^{*}\right)=G_{1}-\left[\pi\left(q_{m}^{*} ; \varepsilon, g_{m}\right)-\pi\left(q_{m}^{*} ; \varepsilon, g_{e}\right)\right]
$$

which is smaller than that in Case 1 and in society under elite rule.

Case 3: Large Outside Opportunities $\left(\varepsilon>\varepsilon^{e}\right)$. There is positive legal investment under both political systems, though legal quality is higher under majority rule than under elite rule: $q_{m}^{*}>q_{e}^{*}>0$. As legal contracts allow agents to exploit new trade opportunities, the traditional advantage of the elite in terms of $g_{e}>g_{m}$ matters less in both societies than before, and the income gap is always lower under majority rule because it can be shown that $G_{2}\left(q_{m}^{*} ; \varepsilon\right)<G_{2}\left(q_{e}^{*} ; \varepsilon\right)$ holds.

32. If the elite agents still use relational contracts, the income gap under majority rule is $G_{1}-\pi\left(q_{m}^{*} ; \varepsilon, g_{m}\right)$. 
An important implication from these scenarios is that as the productivity of new trade opportunities $\varepsilon$ becomes higher relative to that of established partnerships, it is more likely for legal development to start and for legal quality to be higher. Another implication is that legal development often leads to lower income inequality, but legal development is less likely to occur under elite rule than under democracy. So elite rule, lower legal quality, and higher income inequality form an organic cluster of political and legal institutions with corresponding economic outcomes, whereas their opposites, namely democracy, higher legal quality, and lower income inequality, form another cluster.

It is also possible that, under the same political regime, different levels of legal development are caused purely by an arbitrarily small difference in outside opportunities $\varepsilon$. Imagine two identical societies under elite rule. One society experiences a slightly larger shock $\varepsilon=\varepsilon^{e}+u$ and thus invests in legal quality; the other society experiences a slightly smaller shock $\varepsilon=\varepsilon^{e}-u$ and thus does not invest. Even if everything else is identical across the two societies, their economic outlooks are very different: the lucky society has higher legal quality, its agents adopt legal contracts, and its income distribution is more equal than the unlucky one. These results are summarized in the following proposition.

Proposition 4 When outside opportunities $\varepsilon$ are larger, legal development is more likely to start, and it leads to lower income inequality; the process is slower and income inequality is higher under elite rule than under majority rule.

\subsection{Legal Investment with Endogenous Political Systems}

When political dominance has to be backed up by economic strength, it can be shown that the elite are even less willing to invest in legal quality because their relative economic power is likely to be weakened as a result of legal development.

Suppose the political system is determined by the balance of economic power among different groups (Acemoglu and Robinson 2006a). In particular, when the total wealth of the elite agents is higher than that of the masses, the elite group is politically dominant and the society is under elite rule; if the opposite is true, it is under majority rule. The political system is determined both before and after the legal investment decision, since the income distribution may change with legal quality.

When the legal quality is zero, all agents use long-term relational contracts and get corresponding returns $V_{y e}-R_{e}^{*}$ or $V_{y m}-R_{m}^{*}$ in addition to their endowed wealth $w_{e}$ or $w_{m}$; so elite rule happens if

$$
r\left(w_{e}+V_{y e}-R_{e}^{*}\right) \geq(1-r)\left(w_{m}+V_{y m}-R_{m}^{*}\right) .
$$


Elite rule continues automatically if there is no legal reform, since the income distribution remains the same. If the legal quality is increased to $q>0$ and all agents switch from relational to legal contracts, elite rule continues if

$r\left(w_{e}+\hat{V}_{y e}-c_{e}^{*}\right) \geq(1-r)\left(w_{m}+\hat{V}_{y m}-c_{m}^{*}\right)$.

It is easy to show that whenever equation (11) holds, equation (10) will also hold, but the reverse is not true. This means that legal investment weakens the economic power of the elite. To see this more clearly, let $Y(q)$ denote the relative economic power of the elite compared with the masses, where

$Y(q) \equiv r\left(w_{e}+\hat{V}_{y e}-c_{e}^{*}\right)-(1-r)\left(w_{m}+\hat{V}_{y m}-c_{m}^{*}\right)$.

Then 11 is equivalent to $Y(q) \geq 0$; that is, elite rule occurs when $Y(q) \geq 0$ while democracy arises otherwise. And thus $Y(q)$ indicates how secure elite rule is.

Proposition 5 Legal development weakens elite rule but solidifies democracy, since $Y^{\prime}(q)<0$. Specifically, legal quality can never reach above $\bar{q}$ under elite rule, where $Y(\bar{q})=0$.

This proposition suggests that elite rule survives only when $q \leq \bar{q}$, and it is more secure when $q$ is lower; in contrast, democracy arises when $q>\bar{q}$ and it is solidified when $q$ is higher. This means that, when the political system is endogenously determined, $\bar{q}$ is the highest possible legal quality that can sustain elite rule. So even if the elite choose to start the legal development process, it is slower than the socially optimal case and may not increase above a certain threshold $\bar{q}$.

The endogenization of political regimes further strengthens the relationship between elite rule, lower legal quality, and higher income inequality by making them mutually reinforcing; it is not only the case that elite rule leads to slower legal development and hence higher income inequality but also true is the opposite direction where higher income inequality leads to elite rule, which completes the self-perpetuating circle. The other cluster of lower income inequality, democracy, and higher legal quality also forms a self-perpetuating circle with mutually reinforcing elements. One can imagine that, if $\varepsilon$ increases over time, it is possible for the institutional circle of elite rule to persist for a long period and then eventually make the transition to the democracy circle of institutions, though the detail is best left for future research.

\section{Extensions and Discussions}

A key insight of this article is on the importance of trading with new partners: only when the outside opportunities $\varepsilon$ become large enough compared with returns in established partnerships, would a society feel it is desirable to invest in legal contract enforcement in order to facilitate the frequent breaking up of old matches and formation of new ones. Another insight is on the conflict of 
interests in legal development; specifically, the traditional rich elite, who enjoy comparative advantages in relational contracts due to better endowment or privileges, often benefit less from a competent legal system than the masses and hence have less incentive to improve legal quality. These two insights are much more robust than the highly simplified model seems to suggest. To illustrate this, this section introduces some extensions of the basic model and discusses possible interpretations of the results.

\subsection{Contract Enforcement with Social Communities}

In the basic model, agents do not have stable connections among each other except for the bilateral contracts between them, which is not true in reality where individuals belong to different communities. This assumption can be relaxed and our main results still go through and are even strengthened. Since social communities tend to be stronger or function better in a collective-oriented culture than in individualistic ones (Greif 1994), the following arguments and results about the effects of social communities also apply to a collective culture.

To illustrate the main idea, suppose there are $N_{e}$ social communities within the elite group and $N_{m}$ communities among the masses, where $N_{i}>1$ is a finite integer for $i \in\{e, m\}$. A social community could be a family, a kinship network, a clan, an ethnic group, a village or town, or a social club. Members in the same community have formed intricate social connections or developed valuable public goods before the game in the basic model starts. As a result, if a member cheats in the PD as described in Table 1, his partner or the community can impose upon the defector some punishment $x_{i}>0$ at a negligible cost, where $i \in\{e, m\}$. The punishment can take many forms. For example, in a well-organized social club where members enjoy certain privileges, anybody ever found cheating can be formally expelled from the club or informally shunned by other members (Bernstein 1992); neighbors in a small village or members of a close-knit group typically interact with each other in many different ways or even across generations, which provides ample opportunities to punish someone who has cheated (Ellickson 1991).

This means that dealing with a partner from the same community is less risky or less costly than dealing with somebody from outside. It is essentially equivalent to reducing the temptation of cheating in the PD game from $b$ to $b-x_{i}$ for agents in the same community. Another interpretation is that community members have formed multilateral relationships with each other that are worth $x_{i}$ and can be carried on across partnerships. This makes it less costly for agents to break up bilateral relational contracts in order to capture higher gains from trade arising in new partnerships. So in some sense, a part of the benefit of a long-run relationship switches from the bilateral partnership to the community level, where agents can change partners when new matches become more productive but still remain in the multilateral environment of the same community. In other words, the long-term relationship building is now within the borders of a community instead of the much narrower bilateral relations between two individuals. As a consequence, trade efficiency will be enhanced. 
There is, however, a potential drawback for contract enforcement in communities because it makes agents reluctant to trade with outsiders, which becomes more relevant when new partnerships are more productive between agents from different communities than those within the same community. A competent legal system is thus needed to facilitate trading among agents across communities.

Suppose in every period the gain from trade is $a$ with probability $\rho$ in all new partnerships, whereas with probability $1-\rho$ the gain from trade is $a(1+\varepsilon)$ as before for new partnerships within the same community but increases to $a(1+\varepsilon+\theta)$ for those across different communities, where $\theta>0$ indicates the extra gain in productivity from matching with strangers. Similar arguments as in the basic model can be used to show that only when $\theta$ is large enough will legal investment start. Furthermore, if $x_{e} \geq x_{m}$ so that the elite agents are better at enforcing multilateral relationships in communities, they will be even less willing to invest in legal quality than in the basic model. The relevant results are summarized in the following proposition.

Proposition 6 The existence of social communities improves trading efficiency over bilateral relational contracts by reducing relationshipbuilding costs and by encouraging new partnership formation within communities. However, it reduces the incentives of a society to invest in legal quality, the more so when communities are more effective in enforcing relational contracts $\left(x_{i}\right.$ larger), regardless of political regimes.

This proposition suggests that, when social communities are more effective in contract enforcement (when $x_{e}$ or $x_{m}$ is higher), a larger productivity gap between matches within the community and those with outsiders is needed for a society to start legal investment, and the gap is also larger than that in the basic model. So legal development is likely to be slower when social communities are functioning better in facilitating relational contracts regardless of political regimes, which also applies to the socially optimal result. That is, the existence of stronger communities or collective cultures itself does not necessarily lead to inefficiency, though it does cause slower legal development.

These results seem to be useful in understanding differences between East Asia and the West, where social communities are arguably more prevalent in East Asian countries than in the West; and probably as a result of this difference, legal contract enforcement is used less intensively in East Asia than in the West. ${ }^{33}$ This, however, does not necessarily mean that legal development in East Asia is less efficient, which seems to be supported by recent strong

33. It is "commonly acknowledged that East Asian organizations or firms tend to place less emphasis on the use of written, or formal, legal contracts than their western counterparts" (Choi 1994). In Southeast Asia, ethnic Chinese originated from the same region in China usually conduct business transactions in informal clubs (Landa 1981). Generally, networks of interpersonal relationships, based on the traditional kinship emphasis, have played a significant role in East Asia's economic development. Such cultural stress on the collectivity rather than the individual, and the communitarian values and practices are well observed and acknowledged (Huntington 1996). 
economic growth of East Asian countries. In other words, as long as income inequality is not high and political power is not centralized to the elite, a heavier reliance on relational contracts due to competent social communities or cultural preferences does not have to block economic development.

\subsection{Open Versus Closed Society}

In the model, the importance of outside opportunities $\varepsilon$ is taken as exogenously given. This, however, can be readily endogenized to generate new insights. For example, one can imagine that in a closed society that has little contact with the outside world, the outside opportunities $\varepsilon$ tend to be rare and of small scale. According to the arguments in the model, legal investment is less likely to start (due to $\varepsilon<\varepsilon^{i}$ ), and so elite rule is easier to maintain. The opposite is true in an open society, where legal development is likely to start early and eventually weaken elite rule.

Foreseeing such consequences, a society under elite rule would be less willing to adopt an open policy than those under democracy if the degree of openness can be affected by policies, and as a result, it is less likely to experience large outside opportunities and to feel the necessity to improve its legal system. If the political rent is large, which tends to happen when land and other natural resources are abundant, the elite are more likely to adopt a closed-society policy in order to preserve elite rule, and as a consequence, legal development is further retarded.

\subsection{Relevant Examples}

In an effort to understand the development of legal contract enforcement and why it differs across societies, this article uses a theoretical model where legal quality of contract enforcement is a costly public good. This modeling assumption leads to two main results. First, a society would start to invest in legal enforcement only when the benefits of doing so become high enough relative to the default choice of using relational contracts, which happens, for example, when trading with new partners yields higher returns than staying with old ones. Second, when the benefits of improving legal quality of contract enforcement differ across individuals, the actual investment is likely to be affected by political economy forces; in particular, if the traditional elite have comparative advantages over the masses in utilizing relational contracts, legal development may be delayed when the elite hold dominant political power compared with the socially optimal level, whereas the same logic suggests that overinvestment may occur when the masses are in power.

To see whether the insights developed here are useful, it would be desirable to systematically examine the comparative history of contract law development, but such a task is clearly beyond the scope of this article. What this section does is thus very modest; it attempts to convince the reader that the main results in this article are relevant in accounting for some stylized historical facts.

From early medieval times, trade and commerce started to gather momentum in Europe. For several hundred years since then, merchants had to rely on 
social relations and networks (relational contracts) to handle contract issues with each other (Benson 1989). Legal development in contract enforcement was not put on the political agenda until much later in history. For much of the history of the common law in England, for example, contract law remained poorly developed until the law merchant (a medieval series of customs and principles used to regulate trading) was incorporated into the common law under the leadership of Mansfield in the 18th century. ${ }^{34}$

The development of commercial law in England seems to be lagging behind the commercial need; a possible reason proposed in this article is the lack of interest by the ruling monarch and the landed elite class. This becomes evident when one notices that land law was well developed early on and dominated English law, especially in the common law courts. Such a stark contrast is not surprising because, compared with relationships concerning land, other kinds of legal relationships, including commercial contracts and tort, were of little consequence for the landed elite (Zywicki 2003). ${ }^{35}$ Only after commercial and industrial activities became important enough in the economy, were their interests reflected by the political regime change (the Glorious Revolution in 1688 established the political dominance of parliament), which preceded the legal reform in the 18th century that developed the commercial law (Atiyah 1979). ${ }^{36}$

34. It is useful to note that the law merchant, though having its own courts with judges or arbitrators, eventually relies on a merchant's concern of his own reputation and the social network to enforce cooperation. So in essence it is a function of social community (similar to a village's committee of senior people in settling disputes among villagers), and thus still belongs to the realm of relational contracts. If one agrees that legal contract enforcement is characterized by the ultimate reliance on a state's coercive power for enforcement, then it is natural to see that the law merchant should not be regarded as a form of legal enforcement. Furthermore, the fact that the role of the law merchant greatly diminished in England after it was absorbed into the common law seems to indicate the advantage of legal enforcement relative to the law merchant.

35. To connect with the political economy analysis of the model, note that the elite and the masses are both concerned with contractual exchange (Atiyah 1979), where one difference is that the elite (land owners) are concerned more with land transactions while the masses (if without land) with other types of business exchange (e.g., trading goods or services). So when the landed elite are in power, legal enforcement regarding land transfer is developed earlier than in the socially optimal case, whereas legal development in commercial contract enforcement where landed elite have less to gain is delayed; note that in the socially optimal case the land law could have been established later than the general contract law given that land owners have comparative advantages over others in using relational contracts. This is consistent with the general result of the political economy model in the article, which predicts that legal development is often suited to the interests of the politically dominant group; there is, indeed, a minor difference, where in the model the legal quality is assumed to be homogeneous in all contractual activities, whereas in the England case the legal quality of land transactions was different from that of commercial trades. One should bear in mind, however, that this description of English legal development may not tell us the entire story; for example, there are many other differences between the elite and the masses besides their distinct returns from legal enforcement, and as such the body of law related to land that provided peace and security also addressed issues of property rights and some of what now appears in tort law rather than the body of law about commercial affairs per se.

36. As another example, France became the first modern Continental nation at the end of the 15 th century, but there was no unified national body of law (the French civil code of 1804) until after the French Revolution. 
The influence of Atlantic trade on institutional change across Europe (Acemoglu et al. 2005) is also consistent with the main results in this article. The opening of the sea routes to the New World, Africa, and Asia and colonial expansion can be regarded as an exogenous increase in outside trading opportunities $\varepsilon$ in the model. It enriched merchants and traders outside the royal circle and landed elite in England and the Netherlands, and hence altered the balance of political power and prompted corresponding institutional changes that favor commercial interests. However, in countries with absolutist political regimes such as Spain, Portugal, and to some extent France, similar reforms did not happen partially because Atlantic trade was restricted to the royal circle and thus the commercial class did not gain much power during the process. These observations suggest that the presence of large outside opportunities, by altering the political balance of power, is often the ultimate driving force behind legal and other institutional changes; this, however, is less likely to happen in societies where the elite are more dominant.

This article's results may also be useful in understanding differences in legal development between Latin America, East Asia, and the advanced Western countries. The article shows that relational contracts are more widespread when the legal system is of low quality, which is in turn linked to high-income inequality, elite rule, and strong social networks. All of these elements are present in Latin America, whereas the opposites are often observed in the developed West, especially North America, and somewhat in between are East Asian countries under the influence of Confucian culture. ${ }^{37}$

The sharp contrast between Latin and North America may be deeply rooted in their colonial institutions, which in turn can be linked to different levels of inequality in endowment (Engerman and Sokoloff 2002). Indeed, large plantation agriculture and slavery in mining in Latin America induced huge disparities in wealth and thus made it more prone to elite rule and slow legal development. "In contrast, small, family-sized farms were the rule in the northern colonies of the North American mainland, where ... the circumstances fostered relatively homogeneous populations with relatively

37. The empirical evidence (Botero et al. 2004) seems to be consistent with the main results of the model. Legal quality (as negatively indicated by "log number of days to start a business") is lowest in Latin America and highest in the West (including Norway, Denmark, Sweden, Finland, Switzerland, Germany, Austria, Netherlands, France, Belgium, Italy, Spain, Greece, Portugal, US, UK, Canada, Australia, Ireland, Israel, New Zealand), whereas East Asia (including Japan, Korea, Hong Kong, Singapore, Taiwan) is in the middle. The usage of informal contracts as indicated by "size of the unofficial economy" as a percentage of GDP is highest in Latin America and lowest in the West, whereas again East Asia falls in the middle. The per capita GNP and the average schooling level during 1995-2000, which may reflect the extent of economic development, are the lowest in the Latin America, while those of others are much higher and similar to each other. The average Gini index is around 32-36 for both the West and East Asia, while it is 51.4 in Latin America, which has almost the highest income inequality among all countries (United Nations 2005). 
equal distributions of human capital and wealth" (Engerman and Sokoloff 2002). ${ }^{38}$ In both Latin American and East Asian countries, relational contracts are more extensively used, and social communities and collective culture are stronger relative to North America; ${ }^{39}$ a crucial difference between them, however, is that income inequality is much higher in Latin America and elite rule is stronger, which may lead to its lower economic growth and legal quality relative to East Asia. Finally, differences in contract enforcement between East Asia and the West are probably due to cultural differences, and both styles may be justifiable in terms of social welfare optimization. In summary, strong social communities or a collective culture (in East Asia) may slow down legal development (compared with the West) but not necessarily reduce overall welfare; elite rule, however, may cause both (in Latin America), and hence seems to be more detrimental to development.

\section{Conclusions}

Contract enforcement institutions are important for economic performance because most economic exchanges are subject to risk of default and the potential gains from trade may not be realized. This article analyzes the differences between legal contracts and relational contracts at both individual and communal levels and finds that a fundamental conflict of interest in legal investment lies in the different returns of using relational and legal contracts across agents, where the traditional rich elite gain relatively less from legal enforcement than the masses. In other words, it is the less well-endowed masses that stand to gain relatively more from investing in a competent legal system. And so it is not surprising that, if the rich elite are politically dominant and choose legal investment to maximize their own welfare, legal development tends to be slower than in the socially optimal case, whereas overinvestment in legal quality and underusage of relational contracts may happen under majority rule.

Furthermore, it turns out that elite rule, slow legal development, and highincome inequality may form a self-perpetuating circle: Elite rule leads to slow legal development, whereas lower legal quality helps preserve high-income

38. The main contrast between North America and Latin America holds despite some exceptions. Large plantations, for example, existed in the South, but the size of the slave plantations, the share of the population composed of slaves, and the degree of inequality in these colonies were quite modest by the standards of Brazil or the sugar islands. As an exception in Latin America, Argentina was a grain producer, yet by the second half of the 19th century, substantial inequality in the distributions of land, human capital, and political influence was clearly apparent.

39. Generally, contract law involving the sale of goods has become highly standardized in the US as a result of the widespread adoption of the Uniform Commercial Code (UCC) (except in Louisiana), though there is still significant diversity in the interpretation of other kinds of contracts, depending upon the extent to which a given state has codified its common law of contracts or adopted portions of the Restatement (Second) of Contracts (Barnett 2010). These differences are more in the technical details rather than in the quality of legal enforcement. 
inequality, which in turn tends to give rise to elite rule; this suggests that these economic, political, and legal conditions belong to an organic cluster of institutions that generate and support each other in a mutually reinforcing way. It is straightforward to see that the opposite cluster of low-income inequality, democracy, and high legal quality is also self-perpetuating. The transition between these two clusters is an intriguing topic that is worth pursuing in future research.

This article also finds that better functioning social communities help improve trade efficiency in relational contracts and thus may also slow down legal development. This by itself, however, is not necessarily welfare reducing, since legal enforcement is only one alternative among many and its low usage can be the socially optimal result of having better alternatives. Following the same logic as above, only when agents who belong to communities with more effective contract enforcement dictate legal investment decisions, would the resulting lower legal quality be suboptimal. The enforcement quality of social communities is taken as exogenous in this article, presumably as a side-effect of other purposes served by communities. It might be useful in future research to study the formation and function of communities in order to understand how their contract enforcement capabilities, in a broad sense, are endogenously generated.

\section{Appendix}

Proof of $R_{i} \geq b / \delta-a g_{i}+(1-\rho) a \varepsilon$.

Proof. The no-deviation case in an established match is similar. Suppose in the $n$th period the partnership is still not broken, where $n \geq 2$. If an agent cooperates in the PD game, he gets a payoff $a\left(1+g_{i}\right) /(1-\delta)$; if he defects, his payoff is $a\left(1+g_{i}\right)+b+\delta E V_{i}$. So he will not defect if $a\left(1+g_{i}\right) /$ $(1-\delta) \geq a\left(1+g_{i}\right)+b+\delta E V_{i}$, which leads to the same condition (1) as in a new match. The reason is that the benefit of cheating remains the same in both cases.

The possible one-shot deviations when the initial gain from trade is $a(1+\varepsilon)$ can also be analyzed similarly, where deviation is not profitable when (1) is satisfied.

\section{Proof of Lemma 2.}

Proof. The proof focuses only on elite agents, since the mechanism is identical for others. The value of a new match with return $a$ is denoted by $\hat{V}_{y e}$ on the equilibrium path, whereas the value of an established match with return $a\left(1+g_{e}\right)$ is denoted by $\hat{V}_{z e}$. The value of starting a new match with the gain from trade $a(1+\varepsilon)$ is denoted by $\hat{V}_{n e}$ while $c_{n e}$ is the legal cost. When players cooperate in a new match, they get $a$ immediately, followed by a continuation value

$$
E \bar{V}_{e}=\rho \hat{V}_{z e}+(1-\rho)\left(\hat{V}_{n e}-c_{n e}\right),
$$


where with probability $\rho$ agents stay in the same match and get a value $\hat{V}_{z e}$, while with probability $1-\rho$ the old match dissolves as a result of the positive productivity shock $\varepsilon$ and thus agents form a new match with others to get $\hat{V}_{n e}-c_{n e}$. That is,

$\hat{V}_{y e}=a+\delta E \bar{V}_{e}$,

and $\hat{V}_{n e}=a(1+\varepsilon)+\delta E \bar{V}_{e}$. It is easy to see that

$\hat{V}_{n e}=\hat{V}_{y e}+a \varepsilon$.

The continuation value $\hat{V}_{z e}$ is determined in a similar way; the only difference is that the current return is $a\left(1+g_{e}\right)$ due to learning-by-doing in the same match: $\hat{V}_{z e}=a\left(1+g_{e}\right)+\delta E \bar{V}_{e}$. So it is obvious that

$\hat{V}_{z e}=\hat{V}_{y e}+a g_{e}$.

From equations (12)-(14), we get

$\hat{V}_{y e}=\frac{a+\delta \rho a g_{e}+\delta(1-\rho)\left(a \varepsilon-c_{n e}\right)}{1-\delta}$.

Let's check the possible one-shot deviation when the initial gain from trade is $a$. In a new match, if an agent cooperates, the match will continue where he gets $\hat{V}_{y e}-c_{e}$. If he cheats, he gets a payoff of $(a+b)\left(1-Q\left(c_{e}, q\right)\right)+$ $\delta E \hat{V}_{e}-c_{e}$, where the first term is his expected current payoff, and $E \hat{V}_{e}$ is the continuation value in the next period when he becomes an unmatched player since his partner will break up the partnership according to the contract. The expected value of entering a new match is

$E \hat{V}_{e}=\rho\left(\hat{V}_{y e}-c_{e}\right)+(1-\rho)\left(\hat{V}_{n e}-c_{n e}\right)$,

since the net value of starting a new match when the gain from trade is $a$ is $\hat{V}_{y e}-c_{e}$, which occurs with probability $\rho$, while with probability $1-\rho$ the new match is more productive and yields a net value $\hat{V}_{n e}-c_{n e}$. Cheating is thus not optimal when $(a+b)\left(1-Q\left(c_{e}, q\right)\right) \leq \hat{V}_{y e}-\delta E \hat{V}_{e}$ holds, which is simplified to

$(a+b) Q\left(c_{e}, q\right)+\delta \rho c_{e} \geq b-\delta \rho a g_{e}$.

In the $n$th period when the partnership is still not broken, where $n \geq 2$, the oneshot deviation is less profitable than in the first period. If an agent cooperates in the PD game, he gets a payoff $\hat{V}_{z e}$; if he defects, his payoff is $\left[a\left(1+g_{e}\right)+b\right]$ $\left(1-Q\left(c_{e}, q\right)\right)+\delta E \hat{V}_{e}$. So he will not defect if

$\left(a\left(1+g_{e}\right)+b\right) Q\left(c_{e}, q\right)+\delta \rho c_{e} \geq b-\delta \rho a g_{e}$,

which is satisfied when equation (16) holds.

Define $c_{e}^{*}$ to make the equality in equation (16) hold and we get 
$c_{e}^{*}=b / \delta \rho-a g_{e}-(a+b) Q\left(c_{e}^{*}, q\right) / \delta \rho$,

which is the minimum cost of using the legal contract when the legal quality is $q$ and the initial gain from trade is $a$. Based on equation (17), we get

$\frac{\partial c_{e}^{*}}{\partial q}=-\frac{(a+b) Q_{2}}{(a+b) Q_{1}+\delta \rho}<0$,

$\frac{\partial c_{e}^{*}}{\partial g_{e}}=-\frac{\delta \rho a}{(a+b) Q_{1}+\delta \rho}<0$,

and $\partial c_{e}^{*} / \partial \varepsilon=0$.

Proof of Lemma 3.

Proof. The main task of the proof is to calculate the optimal legal $\operatorname{cost} c_{n i}^{*}$, which is uniquely determined by

$\left(a\left(1+g_{i}\right)+b\right) Q\left(c_{n i}^{*}, q\right)+\delta \rho c_{i}^{*}=b-\delta \rho a g_{i}$.

We focus on solving $c_{n e}$ for elite agents, since the mechanism is identical for nonelite agents.

Let's check the possible one-shot deviation when the initial gain from trade is $a(1+\varepsilon)$. In a new match, if an agent cooperates, the match will continue and so he gets $\hat{V}_{n e}-c_{n e}$. If he cheats, he gets a payoff of $(a(1+\varepsilon)+b)$ $\left(1-Q\left(c_{n e}, q\right)\right)+\delta E \hat{V}_{e}-c_{n e}$. Similar exercises as in the text show that cheating is not optimal when $c_{n e} \geq c_{L e}$, where $c_{L e}$ is uniquely determined by $(a(1+\varepsilon)+b) Q\left(c_{L e}, q\right)+\delta \rho c_{e}^{*}=b-\delta \rho a g_{e}$, or equivalently

$Q\left(c_{L e}, q\right)=\frac{a+b}{a(1+\varepsilon)+b} Q\left(c_{e}^{*}, q\right)$.

So $c_{L e}$ is the minimum cost of using the legal contract to deter cheating in a new match when the initial gain from trade is $a(1+\varepsilon)$.

Similar arguments suggest that cheating is not profitable in any $n$th period of a match where the initial gain of trade is $a(1+\varepsilon)$ if

$$
\left(a\left(1+g_{e}\right)+b\right) Q\left(c_{n e}, q\right)+\delta \rho c_{e}^{*} \geq b-\delta \rho a g_{e}
$$

holds. So the minimum legal cost that deters cheating in an established match is $c_{n e}^{*}$ which is determined by

$$
\left(a\left(1+g_{e}\right)+b\right) Q\left(c_{n e}^{*}, q\right)+\delta \rho c_{e}^{*}=b-\delta \rho a g_{e}
$$

or equivalently

$Q\left(c_{n e}^{*}, q\right)=\frac{a+b}{a\left(1+g_{e}\right)+b} Q\left(c_{e}^{*}, q\right)$.

It is easy to check that $c_{n e}^{*}<c_{e}^{*}$ is true, and $c_{n e}^{*}>c_{L e}$ holds if $\varepsilon>g_{e}$. So when the initial gain of trade is $a(1+\varepsilon)$, the legal cost should be at least as large as $c_{n e}^{*}$ to deter cheating when $\varepsilon>g_{e}$. Now we show $c_{n e}^{*}$ also decreases in $q$. Let a new value $\hat{c}$ satisfy $\left(a\left(1+g_{e}\right)+b\right) Q(\hat{c}, q)+\delta \rho \bar{c}=b-\delta \rho a g_{e}$ where $\bar{c}$ is 
a constant such that $\bar{c}>c_{e}^{*}$. Comparing it with equation (20) that determines $c_{n e}^{*}$, we get $c_{n e}^{*}>\hat{c}$. So $c_{n e}^{*}$ is in between $c_{e}^{*}$ and $\hat{c}$, and $c_{n e}^{*}$ becomes identical to $c_{e}^{*}$ when $g_{e}=0$ and arbitrarily close to $\hat{c}$ when $\bar{c}$ is approaching $c_{e}^{*}$. Since both $c_{e}^{*}$ and $\hat{c}$ are decreasing in $q$, so is $c_{n e}^{*}$.

The net value of starting a new match is $\hat{V}_{n e}-c_{n e}$ when the initial return is $a(1+\varepsilon)$, while the value of continuing with the old match for another period is $\hat{V}_{z e}$. So it is optimal for agents to dissolve the old match when $\hat{V}_{n e}-c_{n e} \geq \hat{V}_{z e}$ holds, which leads to $c_{n e}^{*} \leq a\left(\varepsilon-g_{e}\right)$, and thus $\varepsilon_{e}=g_{e}+c_{n e}^{*} / a$.

Next, we prove $\varepsilon_{e}<b / \delta \rho a$. This is indeed so because equations (17) and (20) imply that $\delta \rho c_{n e}^{*}<\delta \rho c_{e}^{*}$ and $\delta \rho c_{e}^{*} \leq b-\delta \rho a g_{e}$, which together implies that $\delta \rho c_{n e}^{*}<b-\delta \rho a g_{e}$ and hence $\varepsilon_{e} \equiv g_{e}+c_{n e}^{*} / a<b / \delta \rho a$.

And finally we show that $\varepsilon_{e}>\varepsilon_{m}$ holds because $g_{e}>g_{m}$ and

$\frac{\partial \varepsilon_{e}}{\partial g_{e}}=1+\frac{1 \partial c_{n e}^{*}}{a \partial g_{e}}>0$.

Note that

$$
\begin{aligned}
\frac{\partial c_{n e}^{*}}{\partial g_{e}} & =\frac{a+b}{a\left(1+g_{e}\right)+b} Q_{1}\left(c_{e}^{*}, q\right) \frac{\partial c_{e}^{*}}{\partial g_{e}}-\frac{a(a+b)}{\left(a\left(1+g_{e}\right)+b\right)^{2}} Q\left(c_{e}^{*}, q\right) \\
& =-\frac{1}{\left[\mathrm{a}\left(1+g_{e}\right)+\mathrm{b}\right]\left[(a+b) Q_{1}+\delta^{*} \rho\right]}-\frac{a(a+b) Q}{\left(a\left(1+g_{e}\right)+b\right)^{2}}<0,
\end{aligned}
$$

where $\frac{\partial c_{e}^{*}}{\partial g_{e}}$ is derived earlier based on equation (17). Let's check whether $\frac{1 \partial c_{n e}^{*}}{a \partial g_{e}}>-1$ or equivalently $\left|\frac{\partial c_{n e}^{*}}{\partial g_{e}} / a\right|<1$ holds; this is indeed true because

$$
\begin{aligned}
\left|\frac{\partial c_{n e}^{*}}{\partial g_{e}}\right| a \mid & <\frac{1}{a\left(1+g_{e}\right)+b}+\frac{a+b}{\left(a\left(1+g_{e}\right)+b\right)^{2}} \\
& =\frac{a\left(1+g_{e}\right)+b+a+b}{\left(a\left(1+g_{e}\right)+b\right)^{2}}<1,
\end{aligned}
$$

where the first inequality holds due to $\frac{(a+b) Q_{1}}{(a+b) Q_{1}+\delta \rho} \delta \rho<1$ and $Q \leq 1$.

Proof of Lemma 4.

Proof. Comparing the legal $\operatorname{cost} c_{e}^{*}$ and the relationship building $\operatorname{cost} R_{e}^{*}$, we get

$R_{e}^{*}-c_{e}^{*}=(1-\rho)(a \varepsilon-b / \delta \rho)+(a+b) Q\left(c_{e}^{*}, q\right) / \delta \rho$.

Comparing the values of these two types of contracts, we get

$$
\hat{V}_{y e}-V_{y e}=\frac{\delta(1-\rho)\left(a \varepsilon-a g_{e}-c_{n e}^{*}\right)}{1-\delta} .
$$

Plugging these two terms in $\pi\left(q ; \varepsilon, g_{e}\right)$, we have

$$
\begin{aligned}
\pi\left(q ; \varepsilon, g_{e}\right) \equiv & \hat{V}_{y e}-c_{e}^{*}-\left(V_{y e}-R_{e}^{*}\right)=\hat{V}_{y e}-V_{y e}+R_{e}^{*}-c_{e}^{*} \\
& =(1-\delta)^{-1}\left[(1-\rho) a \varepsilon-(1-\delta \rho) a g_{e}\right. \\
& \left.-\delta(1-\rho) c_{n e}^{*}\right]-c_{e}^{*}+(1-\rho) b \delta^{-1} .
\end{aligned}
$$


It is straightforward to see that $\partial \pi\left(q ; \varepsilon, g_{e}\right) / \partial \varepsilon=(1-\delta)^{-1}(1-\rho) a>0$ holds as both $c_{e}^{*}$ and $c_{n e}^{*}$ are independent of $\varepsilon$. And

$$
\begin{aligned}
\frac{\partial \pi\left(q ; \varepsilon, g_{e}\right)}{\partial q} & =\frac{\partial}{\partial q}\left[\frac{\delta(1-\rho)\left(a \varepsilon-a g_{e}-c_{n e}^{*}\right)}{1-\delta}+R_{e}^{*}-c_{e}^{*}\right] \\
& =-\frac{\delta(1-\rho) \partial c_{n e}^{*}}{1-\delta}-\frac{\partial c_{e}^{*}}{\partial q}>0
\end{aligned}
$$

holds since both $c_{e}^{*}$ and $c_{n e}^{*}$ are decreasing in $q$ while $R_{e}^{*}$ is independent of $q$. Finally,

$\frac{\partial \pi\left(q ; \varepsilon, g_{e}\right)}{\partial g_{e}}=\frac{\partial\left(\hat{V}_{y e}-V_{y e}\right)}{\partial g_{e}}+\frac{\partial\left(R_{e}^{*}-c_{e}^{*}\right)}{\partial g_{e}}<0$

is true because $\partial\left(R_{e}^{*}-c_{e}^{*}\right) / \partial g_{e}=(a+b) Q_{1}\left(c_{e}^{*}, q\right)(\delta \rho)^{-1} \frac{\partial c_{e}^{*}}{\partial g_{e}}<0$ and

$\frac{\partial\left(\hat{V}_{y e}-V_{y e}\right)}{\partial g_{e}}=-\frac{\delta(1-\rho) a}{1-\delta}\left(1+\frac{1 \partial c_{n e}^{*}}{a \partial g_{e}}\right)<0$

due to equation (21).

\section{Proof of Proposition 1.}

Proof. The first order condition (FOC) for interior solutions is

$$
(1-r) \pi_{1}\left(q^{s} ; \varepsilon, g_{m}\right)+r \pi_{1}\left(q^{s} ; \varepsilon, g_{e}\right)-C^{\prime}\left(q^{s}\right)=0
$$

where

$$
\pi_{1}\left(q ; \varepsilon, g_{e}\right)=-\frac{\delta(1-\rho) \partial c_{n e}^{*}}{1-\delta}-\frac{\partial c_{e}^{*}}{\partial q}>0
$$

and $\pi_{1}\left(q ; \varepsilon, g_{m}\right)$ is similar. The second order condition

$$
\mathrm{SOC} \equiv r \frac{\partial^{2} \pi\left(q ; \varepsilon, g_{e}\right)}{\partial q^{2}}+(1-r) \frac{\partial^{2} \pi\left(q ; \varepsilon, g_{m}\right)}{\partial q^{2}}-C^{\prime \prime}(q)<0
$$

is satisfied because it can be shown that

$$
\frac{\partial^{2} \pi\left(q ; \varepsilon, g_{i}\right)}{\partial q^{2}}=-\frac{\delta(1-\rho) \partial^{2} \tilde{c}_{L i}}{1-\delta \partial q^{2}}-\frac{\partial^{2} \hat{c}_{L i}}{\partial q^{2}}<0 .
$$

So $q^{s}$ is uniquely determined by equation (22). Based on it, we get $\partial q^{s} / \partial \varepsilon=0$ and

$$
\frac{\partial q^{s}}{\partial g_{e}}=\left[r \frac{\partial^{2} \pi\left(q ; \varepsilon, g_{e}\right)}{\partial q \partial g_{e}}+(1-r) \frac{\partial^{2} \pi\left(q ; \varepsilon, g_{m}\right)}{\partial q \partial g_{m}}\right](-\mathrm{SOC})^{-1}<0
$$

where it can be shown that 
$\frac{\partial^{2} \pi\left(q ; \varepsilon, g_{e}\right)}{\partial q \partial g_{e}}=-\frac{\delta(1-\rho) \partial^{2} c_{n e}^{*}}{1-\delta \partial q \partial g_{e}}-\frac{\partial^{2} c_{e}^{*}}{\partial q \partial g_{e}}<0$.

Then from equation (5) we get

$\frac{\partial \varepsilon^{s}}{\partial g_{e}}=-\frac{r \pi_{3}\left(q^{s} ; \varepsilon^{s}, g_{e}\right)}{r \pi_{2}\left(q^{s} ; \varepsilon^{s}, g_{e}\right)+(1-r) \pi_{2}\left(q^{s} ; \varepsilon^{s}, g_{m}\right)}>0$,

where $\pi_{3}<0$ and $\pi_{2}>0$ are proved in Lemma 4 . And Similarly $\partial \varepsilon^{s} / \partial g_{m}>0$ holds.

Proof of Proposition 2.

Proof. The FOC for interior solutions is

$\pi_{1}\left(q_{e}^{*} ; \varepsilon, g_{e}\right)-C^{\prime}\left(q_{e}^{*}\right)=-\frac{\delta(1-\rho) \partial c_{n e}^{*}}{1-\delta \partial q}-\frac{\partial c_{e}^{*}}{\partial q}-C^{\prime}\left(q_{e}^{*}\right)=0$.

The second order condition $\partial^{2} \pi\left(q ; \varepsilon, g_{e}\right) \partial q^{2}-C^{\prime \prime}(q)<0$ is also satisfied so that $q_{e}^{*}$ is uniquely determined by equation (7). Based on it we get $\partial q_{e}^{*} / \partial \varepsilon=0$ and

$\frac{\partial q_{e}^{*}}{\partial g_{e}}=\frac{\partial^{2} \pi\left(q ; \varepsilon, g_{e}\right)}{\partial q \partial g_{e}} /\left(-\frac{\partial^{2} \pi\left(q ; \varepsilon, g_{e}\right)}{\partial q^{2}}-C^{\prime \prime}(q)\right)<0$.

Then from equation (6) we get

$\frac{\partial \varepsilon^{e}}{\partial g_{e}}=-\frac{\pi_{3}\left(q_{e}^{*} ; \varepsilon^{e}, g_{e}\right)}{\pi_{2}\left(q_{e}^{*} ; \varepsilon^{e}, g_{e}\right)}>0$

where $\pi_{3}<0$ and $\pi_{2}>0$ are proved in Lemma 4. Since $\pi_{3}<0$ and $g_{e}>g_{m}$, we know $\pi_{1}\left(q_{e}^{*} ; \varepsilon, g_{m}\right)>\pi_{1}\left(q_{e}^{*} ; \varepsilon, g_{e}\right)$, which implies that

$r \pi_{1}\left(q_{e}^{*} ; \varepsilon, g_{e}\right)+(1-r) \pi_{1}\left(q_{e}^{*} ; \varepsilon, g_{m}\right)-C^{\prime}(q)>\pi_{1}\left(q_{e}^{*} ; \varepsilon, g_{e}\right)-C^{\prime}\left(q_{e}^{*}\right)=0$,

where the equality follows from equation (7). Compared with equation (22), this means $q_{e}^{*}<q^{s}$; that is, the legal quality under elite rule is lower than the socially optimal level. Suppose the opposite condition $\varepsilon^{e} \leq \varepsilon^{s}$ holds; then

$$
\begin{aligned}
0= & r \pi\left(q^{s} ; \varepsilon^{s}, g_{e}\right)+(1-r) \pi\left(q^{s} ; \varepsilon^{s}, g_{m}\right)-C\left(q^{s}\right) \text { by }(5) \\
& >r \pi\left(q_{e}^{*} ; \varepsilon^{s}, g_{e}\right)+(1-r) \pi\left(q_{e}^{*} ; \varepsilon^{s}, g_{m}\right)-C\left(q_{e}^{*}\right) \text { since } q^{s} \text { is the maximizer } \\
& \geq r \pi\left(q_{e}^{*} ; \varepsilon^{e}, g_{e}\right)+(1-r) \pi\left(q_{e}^{*} ; \varepsilon^{e}, g_{m}\right)-C\left(q_{e}^{*}\right) \text { if } \varepsilon^{s} \geq \varepsilon^{e} \text { and by } \pi_{2}>0 \\
& >\pi\left(q_{e}^{*} ; \varepsilon^{e}, g_{e}\right)-C\left(q_{e}^{*}\right) \text { since } g_{e}>g_{m} \text { and by } \pi_{3}<0 \\
& =0 \text { by }(6),
\end{aligned}
$$

which is not possible. Thus, $\varepsilon^{e}>\varepsilon^{s}$ must be true.

\section{Proof of Proposition 3.}

Proof. The optimization part and comparative statics are similar to the above proof and can be easily derived based on the proof of Proposition 1 . 
So they are omitted. Since $\pi_{3}<0$ and $g_{e}>g_{m}$, we know $\pi_{1}\left(q_{m}^{*} ; \varepsilon, g_{e}\right)<$ $\pi_{1}\left(q_{m}^{*} ; \varepsilon, g_{m}\right)$, which implies that

$r \pi_{1}\left(q_{m}^{*} ; \varepsilon, g_{e}\right)+(1-r) \pi_{1}\left(q_{m}^{*} ; \varepsilon, g_{m}\right)-C^{\prime}(q)<\pi_{1}\left(q_{m}^{*} ; \varepsilon, g_{m}\right)-C^{\prime}\left(q_{m}^{*}\right)=0$,

where the equality follows from equation (8). Compared with equation (22), this means $q_{m}^{*}>q^{s}$; that is, the legal quality under majority rule is higher than the socially optimal level. Suppose the opposite condition $\varepsilon^{m} \geq \varepsilon^{s}$ holds; then

$$
\begin{aligned}
0= & r \pi\left(q_{m}^{*} ; \varepsilon^{m}, g_{m}\right)+(1-r) \pi\left(q_{m}^{*} ; \varepsilon^{m}, g_{m}\right)-C\left(q_{m}^{*}\right) \text { by }(9) \\
& >r \pi\left(q^{s} ; \varepsilon^{m}, g_{m}\right)+(1-r) \pi\left(q^{s} ; \varepsilon^{m}, g_{m}\right)-C\left(q^{s}\right) \text { since } q_{m}^{*} \text { is the maximizer } \\
& >r \pi\left(q^{s} ; \varepsilon^{m}, g_{e}\right)+(1-r) \pi\left(q^{s} ; \varepsilon^{m}, g_{m}\right)-C\left(q^{s}\right) \text { since } g_{e}>g_{m} \text { and by } \pi_{3}<0 \\
& >r \pi\left(q^{s} ; \varepsilon^{s}, g_{e}\right)+(1-r) \pi\left(q^{s} ; \varepsilon^{s}, g_{m}\right)-C\left(q^{s}\right) \text { if } \varepsilon^{m} \geq \varepsilon^{s} \text { and by } \pi_{2}>0 \\
& =0 \text { by }(5),
\end{aligned}
$$

which is not possible. Thus, $\varepsilon^{m}<\varepsilon^{s}$ must be true.

\section{Proof of Proposition 5.}

Proof. Note that

$$
Y(q) \equiv r\left(w_{e}+\hat{V}_{y e}-c_{e}^{*}\right)-(1-r)\left(w_{m}+\hat{V}_{y m}-c_{m}^{*}\right)
$$

where

$$
\hat{V}_{y i}-c_{i}^{*}=\frac{a+\delta \rho a g_{i}+\delta(1-\rho)\left(a \varepsilon-c_{n i}^{*}\right)}{1-\delta}-c_{i}^{*} \quad \text { for } i \in\{e, m\}
$$

Then

$$
\begin{aligned}
\frac{\partial Y(q)}{\partial q} & =r \frac{\partial\left(\hat{V}_{y e}-c_{e}^{*}\right)}{\partial q}-(1-r) \frac{\partial\left(\hat{V}_{y m}-c_{m}^{*}\right)}{\partial q} \\
& =r\left[-\frac{\delta(1-\rho) \partial c_{n e}^{*}}{1-\delta}-\frac{\partial c_{e}^{*}}{\partial q}\right]+(1-r)\left[\frac{\delta(1-\rho) \partial c_{n m}^{*}}{1-\delta q}+\frac{\partial c_{m}^{*}}{\partial q}\right] \\
& =r \pi_{1}\left(q ; \varepsilon, g_{e}\right)-(1-r) \pi_{1}\left(q ; \varepsilon, g_{m}\right) \text { by }(23) \\
& <r\left[\pi_{1}\left(q ; \varepsilon, g_{e}\right)-\pi_{1}\left(q ; \varepsilon, g_{m}\right)\right] \text { since }-(1-r)<-r \text { and } \pi_{1}\left(q ; \varepsilon, g_{m}\right)>0 \\
& <0 \text { since } g_{e}>g_{m} \text { and } \pi_{13}\left(q ; \varepsilon, g_{e}\right)<0 \text { by }(24) .
\end{aligned}
$$

So $\partial Y(q) / \partial q<0$; that is, $Y(q)$ is less likely to be positive when $q$ is higher.

\section{Proof of Proposition 6.}

Proof. The following conditions can be derived using similar arguments as in section 3.1 and 3.2. If partners belong to the same community, the minimum relationship building cost to maintain a long-term relational contract is

$\hat{R}_{e} \equiv\left(b-x_{e}\right) / \delta-a g_{e}+a(1-\rho) \varepsilon$,

and the cost of a short-term relational contract that automatically breaks up when a new match becomes more productive is

$\tilde{R}_{s e} \equiv\left(b-x_{e}\right) / \delta \rho-a g_{e}$, 
The short-term relational contract is more profitable than the long-term ones when

$\varepsilon>\left(b-x_{e}\right) / \delta \rho a$.

This part of the proof illustrates how to calculate $\tilde{R}_{s e}$, since the other conditions can be obtained following exactly the same steps as in the text. When $\varepsilon>\left(b-x_{e}\right) / \delta \rho a$, it is optimal to change partners when new matches become more productive. If so, the cost of relationship building is different. Following arguments in section 3.2, the value of a newly formed match is

$\tilde{V}_{y e}=a+\delta\left(\rho \tilde{V}_{z e}+(1-\rho)\left(\tilde{V}_{n e}-\tilde{R}_{n e}\right)\right) \equiv a+\delta E V_{s e}$,

where $E V_{s e}$ is the expected continuation value, and

$$
\begin{aligned}
& \tilde{V}_{z e}=a\left(1+g_{e}\right)+\delta E V_{s e}=a g_{e}+\tilde{V}_{y e}, \\
& \tilde{V}_{n e}=a(1+\varepsilon)+\delta E V_{s e}=a \varepsilon+\tilde{V}_{y e} .
\end{aligned}
$$

So we get

$$
\tilde{V}_{y e}=\frac{a+\delta \rho a g_{e}+\delta(1-\rho)\left(a \varepsilon-\tilde{R}_{n e}\right)}{1-\delta} .
$$

Let's check the possible one-shot deviation when the initial gain from trade is $a$. In a new match, if an agent cooperates, the match will continue where he gets $\tilde{V}_{y e}-\tilde{R}_{e}$. If he cheats, he gets a payoff of $\left(a+b-x_{e}\right)+\delta E \tilde{V}_{y e}-\tilde{R}_{e}$. Note that the expected value of entering a new match $E \tilde{V}_{y e}$ is

$$
\begin{aligned}
E \tilde{V}_{y e} & =\rho\left(\tilde{V}_{y e}-\tilde{R}_{y e}\right)+(1-\rho)\left(\tilde{V}_{n e}-\tilde{R}_{n e}\right) \\
& =\tilde{V}_{y e}+(1-\rho) a \varepsilon-\left(\rho \tilde{R}_{y e}+(1-\rho) \tilde{R}_{n e}\right),
\end{aligned}
$$

since the net value of starting a new match when the gain from trade is $a$ is $\tilde{V}_{y e}-\tilde{R}_{y e}$, which occurs with probability $\rho$, while with probability $1-\rho$ the new match is more productive and yields a net value $\tilde{V}_{n e}-\tilde{R}_{n e}$. Cheating will not happen when

$$
\begin{aligned}
\left(a+b-x_{e}\right) & \leq \tilde{V}_{y e}-\delta E \tilde{V}_{y e}=a+\delta \rho a_{e} g_{e}+\delta \rho \tilde{R}_{y e} \\
& \Rightarrow a+b-x_{e} \leq a+\delta \rho a_{e} g_{e}+\delta \rho \tilde{R}_{y e} \\
& \Rightarrow \tilde{R}_{y e} \geq\left(b-x_{e}\right) / \delta \rho-a g_{e} .
\end{aligned}
$$

The same condition can also deter cheating when the initial gain from trade is $a+\varepsilon$. So the minimum cost of using the short-term relational cost is

$\tilde{R}_{s e}=\left(b-x_{e}\right) / \delta \rho-a g_{e}$

when partners belong to the same community. In the basic model, there is no community so that $x_{e}=0$, and thus the short-term relational cost is $R_{s e}=b / \delta \rho-a g_{e}$, which is larger than $\tilde{R}_{s e}$. 
The existence of social communities improves trade efficiencies in several scenarios, which are summarized in the following table.

The benefit of the second case in Table 2 is calculated as follows.

$$
\begin{aligned}
\tilde{V}_{y e}-\tilde{R}_{s e} & =\frac{a+\delta \rho a g_{e}+\delta(1-\rho)\left(a \varepsilon-\tilde{R}_{s e}\right)}{1-\delta}-\tilde{R}_{s e} \\
& =\frac{a\left(1+g_{e}\right)+\delta(1-\rho) a \varepsilon-(1-\delta \rho)\left(b-x_{e}\right) / \delta \rho}{1-\delta}, \\
V_{y e}-R_{e}^{*} & =\frac{a\left(1+g_{e}\right)}{1-\delta}-b / \delta-a \varepsilon(1-\rho), \\
\tilde{V}_{y e}-\tilde{R}_{s e} & -\left(V_{y e}-R_{e}^{*}\right)=\frac{x_{e}}{\delta}+\left(\varepsilon-\frac{b-x_{e}}{\delta \rho a}\right) \frac{a(1-\rho)}{1-\delta} .
\end{aligned}
$$

Using short-term legal contracts enables agents to form partnerships with individuals from different communities in order to capture the higher gains from trade. The net benefit of doing so is

$$
\begin{aligned}
& \tilde{\pi}\left(q ; g_{e}, x_{e}, \theta\right) \equiv \hat{V}_{s y e}-c_{e}^{*}-\left(\tilde{V}_{y e}-\tilde{R}_{s e}\right) \\
& =\frac{\delta(1-\rho)\left[a \theta-c_{n e}^{*}\right]+(1-\delta \rho)\left[\left(b-x_{e}\right) / \delta \rho-a g_{e}\right]}{1-\delta}-c_{e}^{*}
\end{aligned}
$$

for an elite agent, where $\hat{V}_{\text {sye }}$ is the same as $\hat{V}_{y e}$ in equation (15) except by replacing $\varepsilon$ by $\varepsilon+\theta$. It is easy to see that $\partial \tilde{\pi}\left(q ; g_{e}, x_{e}, \theta\right) / \partial x_{e}<0$ holds, which means that the benefit of using legal contracts is lower when $x_{e}$ is higher or when the communities are more effective in enforcing relational contracts.

Let $\tilde{q}^{s}$ denote the socially optimal legal quality and $\theta^{s}$ the threshold level of $\theta$ beyond which legal investment starts. The FOC for interior solutions is

$r \tilde{\pi}_{1}\left(\tilde{q}^{s} ; g_{e}, x_{e}, \theta\right)+(1-r) \tilde{\pi}_{1}\left(\tilde{q}^{s} ; g_{m}, x_{m}, \theta\right)-C^{\prime}\left(\tilde{q}^{s}\right)=0$,

where

$\tilde{\pi}_{1}\left(\tilde{q}^{s} ; g_{e}, x_{e}, \theta\right)=-\frac{\delta(1-\rho) \partial c_{n e}^{*}}{1-\delta \partial q}-\frac{\partial c_{e}^{*}}{\partial q}=\pi_{1}\left(\tilde{q}^{s} ; \varepsilon, g_{e}\right)$

as in the basic model. The second-order condition is also the same as before. So $\tilde{q}^{s}$ is uniquely determined by equation (28). Based on it, we get the following comparative statics:

$\frac{\partial \tilde{q}^{s}}{\partial \theta}=0, \frac{\partial \tilde{q}^{s}}{\partial x_{e}}=0$.

$\theta^{s}$ is determined by

Table 2. Trade Efficiencies Improved by Communities

\begin{tabular}{llll}
\hline Cases & with community & Basic model & Benefit of community \\
\hline$\varepsilon \leq \frac{b-x_{e}}{\delta \rho a}$ & $V_{y e}-\hat{R}_{e}($ long - term $)$ & $V_{y e}-R_{e}^{*}($ long - term $)$ & $\frac{x_{e}}{\delta}$ \\
$\varepsilon \in\left(\frac{b-x_{e}}{\delta \rho a}, \frac{b}{\delta \rho a}\right)$ & $\tilde{V}_{y e}-\tilde{R}_{s e}($ short - term $)$ & $V_{y e}-R_{e}^{*}($ long - term $)$ & $\frac{x_{e}}{\delta}+\left(\varepsilon-\frac{b-x_{e}}{\delta \rho a}\right) \frac{a(1-\rho)}{1-\delta}$ \\
$\varepsilon>\frac{b}{\delta \rho a}$ & $\tilde{V}_{y e}-\tilde{R}_{s e}($ short - term $)$ & $\tilde{V}_{y e}-R_{s e}($ short - term $)$ & $\frac{x_{e}}{\delta \rho}$ \\
\hline
\end{tabular}


$r \tilde{\pi}\left(\tilde{q}^{s} ; g_{e}, x_{e}, \theta^{S}\right)+(1-r) \tilde{\pi}\left(\tilde{q}^{S} ; g_{m}, x_{m}, \theta^{S}\right)-C\left(\tilde{q}^{S}\right)=0$,

based on which we get

$\frac{\partial \theta^{s}}{\partial x_{e}}=-\frac{r \tilde{\pi}_{3}\left(\tilde{q}^{s} ; g_{e}, x_{e}, \theta^{s}\right)}{r \tilde{\pi}_{4}\left(\tilde{q}^{s} ; g_{e}, x_{e}, \theta^{s}\right)+(1-r) \tilde{\pi}_{4}\left(\tilde{q}^{s} ; g_{m}, x_{m}, \theta^{s}\right)}>0$,

where $\tilde{\pi}_{3}<0$ and $\tilde{\pi}_{4}>0$ are indeed true as both $c_{e}^{*}$ and $c_{n e}^{*}$ are independent of $\theta$ and $x_{e}$. Similarly $\partial \theta^{s} / \partial x_{m}>0$ holds. And $\theta^{s}>\varepsilon^{s}$ holds because $\tilde{\pi}\left(q ; g_{e}, x_{e}, \theta\right)<\pi\left(q ; \theta, g_{e}\right)$, which is implied by $\tilde{V}_{y e}-\tilde{R}_{s e}>V_{y e}-R_{e}^{*}$.

It is straightforward to see that the same comparative statics hold for both majority rule and elite rule under similar arguments.

\section{Funding}

Singapore Management University research grant (MSS5E007).

\section{References}

Acemoglu, D. 2008. "Oligarchic versus Democratic Societies," 6 Journal of the European Economic Association 1-44.

Acemoglu, D., S. Johnson, and J. Robinson. 2005. "The Rise of Europe: Atlantic Trade, Institutional Change and Economic Growth," 95 American Economic Review 546-79.

Acemoglu, D., and J. Robinson. 2006a. Economic Origins of Dictatorship and Democracy. Cambridge: Cambridge University Press.

2006b. "Economic Backwardness in Political Perspective," 100 American Political Science Review 115-31.

2008. "Persistence of Power, Elites and Institutions," 98 American Economic Review $267-93$.

Adams, J. N., and R. Brownsword. 2007. Understanding Contract Law. 5th ed. London: Sweet \& Maxwell Ltd.

Atiyah, P. S. 1979. The Rise and Fall of Freedom of Contract. New York: Oxford University Press.

Banerjee, A., and A. Newman. 1993. "Occupational Choice and the Process of Development," 101 Journal of Political Economy 274-98.

Barnett, R. E. 2010. The Oxford Introductions to U.S. Law: Contracts. New York: Oxford University Press.

Baye, Michael R., Dan Kovenock, and Casper G. Vries. 2005. "Comparative Analysis of Litigation Systems: An Auction-Theoretic Approach,” 115 Economic Journal 583-601.

Benson, B. L. 1989. "The Spontaneous Evolution of Commercial Law," 55 Southern Economic Journal 644-61.

Bernstein, L. 1992. "Opting out of the Legal System: Extralegal Contractual Relations in the Diamond Industry," 21 Journal of Legal Studies 115-57.

Besley, T., and M. Ghatak. 2009. "The de Soto Effect." Working paper, London School of Economics.

Botero, J., S. Djankov, R. La Porta, F. Lopez-de-Silanes, and A. Shleifer. 2004. "The Regulation of Labor,” 119 Quarterly Journal of Economics 1339-82.

Carmichael, H. L, and W. B. MacLeod. 1997. "Gift Giving and the Evolution of Cooperation," 38 International Economic Review 485-509.

Choi, C. J. 1994. "Contract Enforcement Across Cultures," 15 Organization Studies 673-82.

Chong, A., and M. Gradstein. 2007a. "Inequality and Institutions," 89 Review of Economics and Statistics $454-65$.

2007b. "Inequality and Informality," 91 Journal of Public Economics 159-79. 
Cooter, R., and J. Landa. 1984. "Personal versus Impersonal Trade: The Size of Trading Groups and the Law of Contracts," 4 International Review of Law and Economics 15-22.

De Soto, H. 1989. The Other Path: The Invisible Revolution in the Third World. New York: Harper \& Row.

Dhillon, A., and J. Rigolini. 2011. "Development and the Interaction of Enforcement Institutions," 95 Journal of Public Economics 79-87.

Dixit, A. 2003. "Trade Expansion and Contract Enforcement," 111 Journal of Political Economy 1293-317.

2009. "Governance Institutions and Economic Activity," 99 American Economic Review $5-24$.

Durlauf, S., and M. Fafchamps. 2005. "Social Capital," in S. Durlauf and P. Aghion, eds., Handbook of Economic Growth. Amsterdam: North-Holland.

Ellickson, R. C. 1991. Order without Law. Cambridge: Harvard University Press.

Engerman, S. L, and K. L. Sokoloff. 2002. "Factor Endowments, Inequality, and Paths of Development among New World Economies."NBER Working Paper \#9259.

2005. "The Evolution of Suffrage Institutions in the New World," 65 Journal of Economic History 891-921.

Fafchamps, M. 2002. "Spontaneous Market Emergence," 2 Topics in Theoretical Economics.

Furmston, M. P. 2006. Cheshire, Fifoot and Furmston's Law of Contract. 15th ed. New York, NY: Oxford University Press.

Glaeser, E., J. Scheinkman, and A. Shleifer. 2003. "The Injustice of Inequality," 50 Journal of Monetary Economics 199-222.

Greif, A. 1994. "Cultural Beliefs and the Organization of Society: A Historical and Theoretical Reflection on Collectivist and Individualist Society," 102 Journal of Political Economy $912-50$.

2002. "Institutions and Impersonal Exchange: From Communal to Individual Responsibility," 158 Journal of Institutional and Theoretical Economics 168-204.

2005. "Commitment, Coercion and Markets: The Nature and Dynamics of Institutions Supporting Exchange," in Claude Menard and Mary Shirley, eds., Handbook of New Institutional Economics, 727-786. Netherlands: Kluwer.

2006. Institutions and the Path to the Modern Economy: Lessons from Medieval Trade. Cambridge: Cambridge University Press.

Hadfield, G. K. 2005. "The Many Legal Institutions that Support Contractual Commitment, ” in Claude Menard and Mary Shirley, eds., Handbook of New Institutional Economics, 175-203. The Netherlands: Kluwer.

Harris, R. 2003. "The Encounters of Economic History and Legal History," 21 Law and History Review 297-346.

Hoff, K., and J. E. Stiglitz. 2004. "After the Big Bang? Obstacles to the Emergence of the Rule of Law in Post-Communist Societies," 94 American Economic Review 753-63.

Huntington, S. P. 1996. The Clash of Civilizations and the Remaking of World Order. New York: Simon \& Schuster.

Johnson, S., J. McMillan, and C. Woodruff. 2002. "Courts and Relational Contracts," 18 Journal of Law, Economics and Organization 221-77.

Kranton, R. 1996a. "Reciprocal Exchange: A Self-Sustaining System," 86 American Economic Review 830-51.

1996b. "The Formation of Cooperative Relationships," 12 Journal of Law Economics and Organization 214-33.

Kranton, R., and A. Swamy. 1999. "The Hazards of Piecemeal Reform: British Civil Courts and the Credit Market in Colonial India," 58 Journal of Development Economics 1-24.

Landa, J. T. 1981. "A Theory of the Ethnically Homogeneous Middleman Group: An Institutional Alternative to Contract Law," 10 Journal of Legal Studies 349-62.

Li, S.. 2003. "Relation-Based Versus Rule-Based Governance: An Explanation of the East Asian Miracle and Asian Crisis," 11 Review of International Economics 651-73.

Macaulay, S. 1963. "Non-Contractual Relations in Business: A Preliminary Study," 28 American Sociological Review 55-67. 
MacLeod, W. B. 2007. "Reputations, Relationships, and Contract Enforcement," 45 Journal of Economic Literature 595-628.

Magee, Stephen P., William A. Brock, and Leslie Young. 1989. Black Hole Tariffs and Endogenous Policy Theory. Cambridge: Cambridge University Press.

McMillan, J., and C. Woodruff. 1999. "Interfirm Relationships and Informal Credit in Vietnam," 114 Quarterly Journal of Economics 1285-320.

Murphy, K. M, Andrei Shleifer, and R. W. Vishny. 1991. "The Allocation of Talent: Implications for Growth," 106 Quarterly Journal of Economics 503-30.

North, D. C. 1991. "Institutions,"5 Journal of Economic Perspectives 97-112.

North, D. C., W. Summerhill, and B. R. Weingast. 2000. "Order, Disorder and Economic Change: Latin America vs. North America," in B.B. de Mesquita and H. Root, eds., Governing for Prosperity, 17-58. New Haven: Yale University Press.

North, D. C., J. J. Wallis, and B. R. Weingast. 2009. Violence and Social Orders: A Conceptual Framework for Interpreting Recorded Human History, Cambridge: Cambridge University Press.

Peng, S. 2000. "The WTO Legalistic Approach and East Asia: From the Legal Culture Perspective," 13 Asian-Pacific Law and Policy Journal 1-35.

Pipes, R., 1995. Russia under the Old Regime. 2nd ed. London: Penguin Books.

Portes, A. 1994. "The Informal Economy and Its Paradoxes, " in Neil J. Smelser and Richard Swedberg, eds., Handbook of Economic Sociology. Princeton, NJ: Princeton University Press.

Putnam, R. D. 1995. "Bowling Alone: America's Declining Social Capital," 6 Journal of Democracy 65-78.

Reed, S. R. 2001. "Impersonal Mechanisms and Personal Networks in the Distribution of Grants in Japan.”Working Paper, Chuo University, Japan.

Sobel, J. 2006. "For Better or Forever: Formal versus Informal Enforcement," 24 Journal of Labor Economics 271-97.

Sonin, K. 2003. "Why the Rich May Favor Poor Protection of Property Rights," 31 Journal of Comparative Economics 715-31.

United Nations. 2005. Development Programme Report.

Weber, M. 1954, "On Law in Economy and Society." Trans. E. Shils and M. Rheinstein. Cambridge, MA: Harvard University Press.

Whyte, M. K. 1996. "The Chinese Family and Economic Development: Obstacle or Engine?" 45 Economic Development and Cultural Change 1-30.

Zywicki, T. J. 2003. "The Rise and Fall of Efficiency in the Common Law: A Supply-Side Analysis," 97 Northwestern Law Review 1551-633. 